\title{
Gênero Strombomonas Deflandre (Euglenophyceae pigmentadas em ambiente lótico subtropical, município de Triunfo, RS, Brasil: riqueza e distribuição geográfica
}

\author{
Sandra Maria Alves-da-Silva ${ }^{1,4}$, Josemari da Cruz Cabreira ${ }^{2}$, Karina Federle Consoni $^{2}$ e Eduardo Alexis Lobo ${ }^{3}$ \\ Recebido: 27.12.2012; aceito: 28.05.2013
}

\begin{abstract}
The genus Strombomonas Deflandre (pigmented Euglenophyceae) in a subtropical lotic environment, Municipality of Triunfo, Rio Grande do Sul State, Brazil: richness and geographical distribution). A taxonomic study of the genus Strombomonas Defl. was carried out in three reaches of the stream Bom Jardim, Municipality of Triunfo, Rio Grande do Sul State, expanding the knowledge of its richness and geographical distribution. Samples were collected monthly (2002-2006), with plankton net and preserved with formaldehyde. Simultaneously, water samples for measurement of physicochemical variables were collected. Twenty-six species of Strombomonas were identified; eight taxa have cosmopolitan distribution and the remainder are restricted to a maximum of three continents. The cluster analysis indicated that the greatest similarity occurred between the stream sections T2 and T3, equal to $82 \%$, differently from T1 (upper reaches). This difference can be explained considering that in T1 the exclusive presence of the species S. brevicaudata, S. scabra var. labiata, and S. rotunda was verified. Strombomonas brevicaudata Conf. is registered for the first time in the State. Strombomonas eurystoma (Stein) f. incurva (Buz.) Pop., and S. recurvata Conf. \& Joo are new records for Brazil.
\end{abstract}

Key words: Euglenophyta, shallow stream, Southern Brazil

RESUMO - (Gênero Strombomonas Deflandre (Euglenophyceae pigmentadas) em ambiente lótico subtropical, Município de Triunfo, RS, Brasil: riqueza e distribuição geográfica). O objetivo foi realizar um estudo taxonômico do gênero Strombomonas Deflandre em três trechos do arroio Bom Jardim, Município de Triunfo, RS, ampliando o conhecimento da sua riqueza e distribuição geográfica. As amostras foram coletadas mensalmente (2002-2006), com rede de plâncton e conservadas com formaldeído. Simultaneamente foram realizadas coletas d'água para medição de variáveis físico-químicas. Foram identificadas 26 espécies de Strombomonas, sendo que oito táxons apresentam distribuição cosmopolita e os demais estão restritos no máximo a três continentes. O agrupamento indicou que a maior similaridade ocorreu entre os trechos T2 e T3, igual a $82 \%$, diferentemente de T1 (nascente). Esta diferença explica-se considerando que em T1 houve a presença exclusiva das espécies $S$. brevicaudata, $S$. scabra var. labiata e $S$. rotunda. S. brevicaudata Conf. é citada pela primeira vez para o Estado. S. eurystoma (Stein) f. incurva (Buz.) Pop. e S. recurvata Conf. \& Joo são novos registros para o Brasil. Palavras-chave: arroio raso, Euglenophyta, sul do Brasil

\section{Introdução}

A divisão Euglenophyta é constituída por algas microscópicas, em geral unicelulares (com exceção do gênero Colacium), livre natantes graças a um ou mais flagelos emergentes, inserido na região anterior da célula. Mais de 800 espécies foram citadas por Huber-Pestalozzi (1955), com um total 2.000 espécies estimada por Norton et al. (1996).
Existe carência no estudo desse grupo de algas no país possivelmente pelo número reduzido de especialistas; pelo fato dessas algas apresentarem rápido deslocamento pela presença de flagelo(s); por apresentarem uma película protéica, o que permite algumas espécies mudarem sua forma, fenômeno conhecido como metabolia ou movimento euglenóide. Para seu estudo também é necessário observação de amostras vivas e fixadas o que demanda o dobro de

1. Fundação Zoobotânica do Rio Grande do Sul, Museu de Ciências Naturais, Seção de Botânica de Criptógamas, Caixa Postal 1188, 90690-000 Porto Alegre, Rio Grande do Sul, Brasil

2. Universidade Luterana do Brasil, Curso de Ciências Biológicas, Caixa Postal 124, 92425-900 Canoas, Rio Grande do Sul, Brasil

3. Laboratório de Limnologia, Universidade de Santa Cruz do Sul, Caixa Postal 236, 96815-900, Santa Cruz do Sul, Rio Grande do Sul, Brasil

4. Autor para correspondência: alvesdasilva@fzb.rs.gov.br 
tempo para sua análise. Nas últimas décadas o grupo também tem passado por transformações taxonômicas devido a estudos envolvendo biologia molecular (e.g. Linton et al. 2000, Marin et al. 2003, Brosnan et al. 2003, 2005, Bennett \& Triemer 2012).

As algas, entre as quais as Euglenophyta, possuem grande importância ecológica porque são a base da cadeia trófica, servem de alimento ao zooplâncton, e ao realizarem fotossíntese fornecem oxigênio no meio aquático. Existem muitas espécies dessa divisão que são consideradas excelentes bioindicadoras de águas poluídas por matéria orgânica (Sladecék 1973, Margalef 1983, Round 1983).

O objeto deste estudo foi o gênero Strombomonas Deflandre, classe Euglenophyceae, ordem Euglenales, família Euglenaceae que engloba indivíduos microscópicos, pigmentados, solitários, livre natantes, rodeados por uma carapaça denominada de lórica, com forma variada, lisa ou não. Segundo Conforti et al. (1994), a lórica das Strombomonas apresenta como principais componentes na sua composição a sílica e o ferro. Estudos utilizando microscópio eletrônico de varredura (MEV) têm revelado que a lórica irregular, granulosa, escabrosa ou verrucosa é resultado da aglutinação de partículas exógenas aglutinadas a sua parede (Tell \& Conforti 1988, Conforti 1993, Conforti \& Joo 1994, Conforti \& Zalocar 2003, Wolowski \& Walne 2007).

Possui 50 espécies (Bourrelly 1970), ocorrendo principalmente em águas doces com altos teores de matéria orgânica do mundo inteiro (Hoeck et al. 1995), e em corpos d'água lóticos (Tell \& Conforti 1986). Desde a criação de Strombomonas por Deflandre (1930), a partir de um grupo de espécies de Trachelomonas da subseção Saccatae, série Asperae, a validade do gênero tem sido discutida.

Vários trabalhos envolvendo a composição, desenvolvimento e ultraestrutura da lórica têm sido publicados (e.g. West \& Walne 1980, Dunlap et al. 1986, Conforti et al. 1994, Wang et al. 2003, Brosnan et al. 2005). Destaca-se, entretanto, o trabalho de Ciugulea et al. (2008), o qual permitem concluir pela manutenção da separação dos gêneros Trachelomonas e Strombomonas, com base na morfologia e biologia molecular de táxons desses dois gêneros loricados.

Muitas espécies de Strombomonas apresentam distribuição cosmopolita (Bourrelly 1970), mas a maioria dos táxons desse gênero ocorre em pequeno número de indivíduos por lâminas, o que dificulta estudos populacionais.

No Brasil, trabalhos sobre taxonomia de Euglenophyta têm sido publicados nas últimas décadas
(Menezes \& Fernandes 1987,1989, Alves-da-Silva 1988, Xavier 1988,1993, 1994, 1996, Huzsar et al. 1989, Cecy 1990, Franceschini 1992, Menezes 1992, Alves-da-Silva \& Torres 1994 a,b, Conforti 1994, Jati \& Train 1994, Bittencourt-Oliveira 1997, Ferreira \& Menezes 2000, Keppeler et al. 2002, Alves-da-Silva \& Bicudo 2003, 2006, 2009, Alves-da-Silva \& Bridi 2004, Alves-da-Silva \& Hahn 2005, Alves-da-Silva \& Fortuna 2006, Alves-da-Silva \& von Kurrle 2009, Alves-da-Silva et al. 2011 e Araújo et al. 2012). Entretanto, o volume de informações desse grupo de algas em nível estadual e nacional ainda é bastante escasso, face aos inúmeros ambientes aquáticos existentes no país.

Neste contexto, o presente trabalhou objetivou realizar um estudo taxonômico do gênero Strombomonas, em três trechos do arroio Bom Jardim, Município de Triunfo, Estado do Rio Grande do Sul, no período de 2002 a 2006, ampliando o conhecimento sobre sua riqueza e distribuição geográfica mundial.

\section{Material e métodos}

O arroio Bom Jardim é afluente da margem direita do Rio Caí, no município de Triunfo, com quase todo seu percurso, de $10,5 \mathrm{~km}$, situado na área do Pólo Petroquímico de Triunfo no Estado do Rio Grande do Sul. O arroio localiza-se próximo ao curso inferior do Caí, cujas águas são utilizadas para abastecimento público e industrial, extração de argila, areia e cascalho e diluição de despejos domésticos e industriais. O clima local é subtropical com precipitação pluvial anual média no Estado do Rio Grande do Sul de $1.500 \mathrm{~mm}$, sendo as maiores precipitações totais mensais verificadas em junho e julho (Conti \& Furlan 2003). Nesse arroio foram amostrados três trechos, denominados ABJNascente (T1) (29 49'36"S-51 $\left.27^{\circ} 13^{\prime \prime} \mathrm{W}\right)$, ABJMontante ao SITEL (T2), onde a sigla "SITEL" é a abreviatura de Sistema de Tratamento de Efluentes Líquidos do Pólo Petroquímico de Triunfo (29050'22"S-51 $\left.25^{\circ} 01^{\prime \prime} \mathrm{W}\right)$ e ABJFrente ao SITEL (T3) (2950'13"S-51 $\left.{ }^{\circ} 23^{\prime} 26^{\prime \prime} \mathrm{W}\right)$ (figura 1). A estação ABJNascente é o trecho mais raso do arroio (máximo $30 \mathrm{~cm}$ de profundidade). Possui em seu entorno sítios com gado, ovelhas e pomares; o trecho ABJMontante recebe influência de poluição antrópica de uma vila sem rede de esgotos (máximo $80 \mathrm{~cm}$ de profundidade); e o ABJFrente é o trecho próximo a foz e que possui maior influência do complexo Petroquímico (máximo de $150 \mathrm{~cm}$ de profundidade).

As amostras foram coletadas através de 30-40 arrastos horizontais na subsuperfície da água com 
rede de plâncton de 25 e $30 \mu \mathrm{m}$ de abertura de malha, e conservadas com formaldeído a $4 \%$. As coletas tiveram periodicidade mensal, entre 2002 a 2006. Para a análise qualitativa foram preparadas lâminas temporárias e observadas em média 10 lâminas por amostra, em microscópio Leica DMS, com ocular micrometrada, e realizados desenhos dos táxons com auxílio de câmara-clara acoplada a esse sistema óptico.

Concomitantemente à coleta do material biológico, foi realizada coleta da água para realização de medidas das variáveis ambientais como sílica $\left(\mathrm{mg} \mathrm{L}^{-1}\right)$, matéria orgânica $\left(\mathrm{mg} \mathrm{O}_{2} \mathrm{~L}^{-1}\right)$, oxigênio dissolvido $\left(\mathrm{mg} \mathrm{L}^{-1}\right)$, demanda bioquímica de oxigênio $\left(\mathrm{mg} \mathrm{L}^{-1}\right)$ e ortofosfato $\left(\mu \mathrm{g} \mathrm{L}^{-1}\right)$ que foram medidas no laboratório seguindo as técnicas descritas em APHA (1992). No local foram medidas a temperatura da água e ar $\left({ }^{\circ} \mathrm{C}\right.$, termômetro de mercúrio), $\mathrm{pH}$ (potenciômetro modelo DMPH-P), profundidade $(\mathrm{cm})$ e condutividade elétrica $\left(\mu \mathrm{S} \mathrm{cm}^{-1}\right.$, condutivimetro Digimed, modelo CD-28). Os dados da precipitação pluviométrica foram obtidos do $8^{\circ}$ Distrito de Meteorologia - INMET, Instituto Nacional de Meteorologia do Ministério da Agricultura e do Abastecimento, que possuem uma Estação Meteorológica próxima à área de estudo.

Para verificação da similaridade específica qualitativa entre os três trechos do arroio aplicou-se uma análise de agrupamento (cluster), tendo como base a presença e ausência dos táxons identificados, e utilizando como medida de similaridade o índice de Jaccard (Ludwig \& Reynolds 1988). Trabalhou-se com o programa estatístico PAST (Hammer et al. 2001).

Com relação à frequência de ocorrência $(\mathrm{Fo}=\mathrm{D} .100 / \mathrm{d}$, onde $\mathrm{D}$ é número de amostras em que o táxon foi encontrado e d é número total de amostras), os táxons foram classificados como constantes
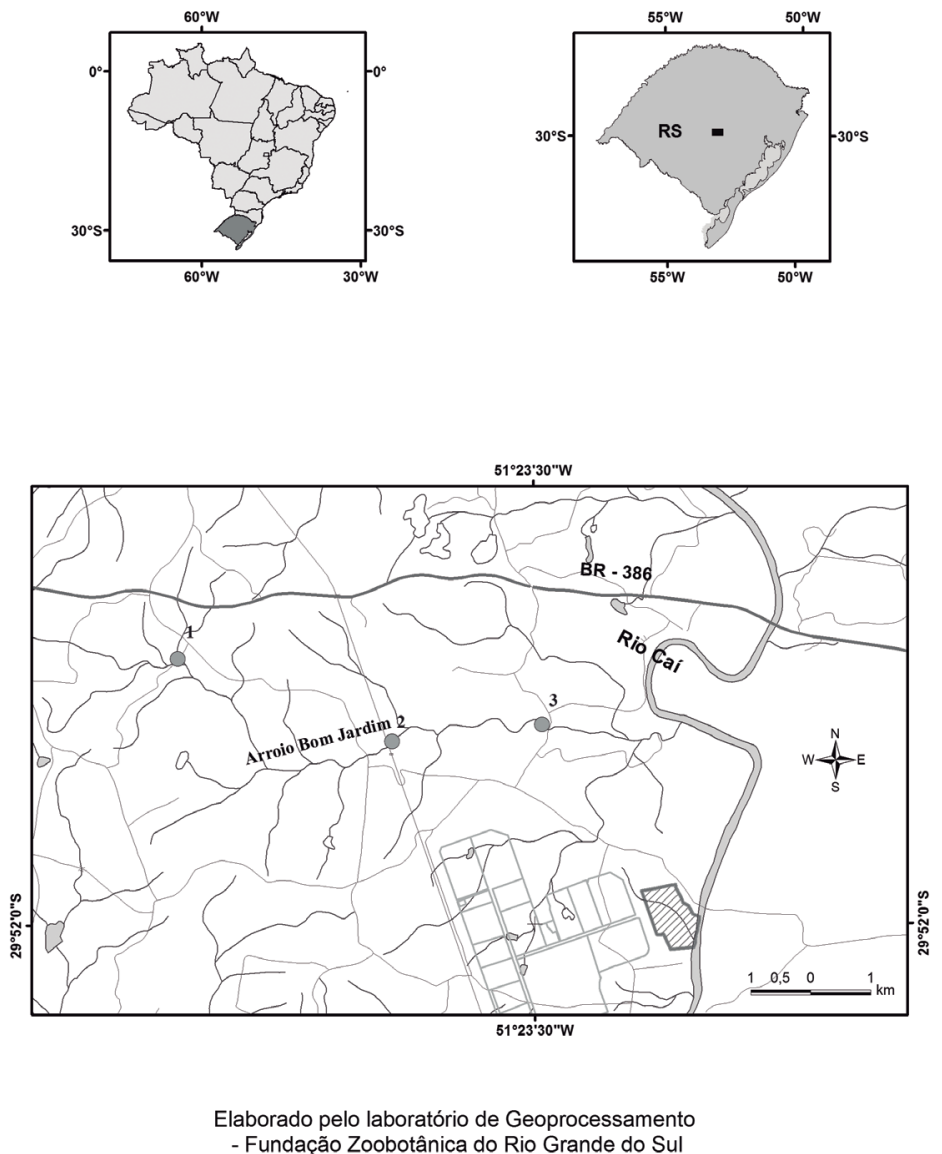

Figura 1. Localização do arroio Bom Jardim, município de Triunfo, RS, Brasil, com a indicação dos três trechos amostrados. T1: Nascente; T2. Montante ao Sitel; T3. Frente ao Sitel.

Figure 1. Location of Bom Jardim Stream, municipality of Triunfo, RS, Brazil, indicating the three sampled reaches. T1: Spring, T2: Upstream of Sitel, T3: Front of Sitel. 
(frequência maior que 50\%), comuns (frequência entre $10 \%$ e $50 \%$ ) e raros (frequência menor que $10 \%$ ).

O trabalho apresenta a descrição completa dos táxons que constituem no primeiro registro para o Estado do Rio Grande do Sul e/ou Brasil. Para todos os táxons, são fornecidas informações quanto à morfologia, amplitude das dimensões, relação entre o comprimento e a largura $(\mathrm{Rc} / \mathrm{l})$, ilustrações, número do lote em que foram registradas as espécies e infraespécies, a distribuição geográfica no país por região e estado, e as variáveis ambientais.

Para a identificação específica e infraespecífica foram utilizadas obras básicas como: Conrad \& Deflandre (1930), Van-Meel (1952), Huber-Pestalozzi (1955), Popova (1966), Starmach (1983), Tell \& Conforti (1986, 1988), Shi et al. (1999) Dillard (2000).

Todos os lotes analisados encontram-se incorporados ao Herbário Prof. Dr. Alarich R.H. Schultz (HAS) do Museu de Ciências Naturais da Fundação Zoobotânica do Estado do Rio Grande do Sul.

\section{Resultados e Discussão}

Foram analisadas 175 amostras e registrado o gênero em 96 delas $(54,9 \%)$, resultando na identificação de 26 táxons específicos e infraespecíficos de Strombomonas, distribuídos entre 16 espécies típicas, sete variedades que não são as típicas e três formas que também não são as típicas.

Gênero Strombomonas Deflandre 1930

Strombomonas borystheniensis (Roll) Pop., Flora plant. Cryptog. 3(1): 206, pl. 20, fig. 15. 1966.

Figura 2

Lórica amplamente elíptica, 25-26 $\mu \mathrm{m}$ compr., ca. $21 \mu \mathrm{m}$ larg., $\mathrm{Rc} / \mathrm{l}=1,2$.

Material examinado: BRASIL. Rio GRANDE do Sul: Triunfo, arroio Bom Jardim T2, 6-II-2002, M.L. Nunes s.n. (HAS103188); Triunfo, arroio Bom Jardim T3, 6-II-2002, M.L. Nunes s.n. (HAS103195); Triunfo, arroio Bom Jardim T1, 6-II-2002, M.L. Nunes s.n. (HAS103202); Triunfo, arroio Bom Jardim T3, 22-IV-2002, S.M. Alves da Silva s.n. (HAS103397); Triunfo, arroio Bom Jardim T2, 2-IX-2002, S.M. Alves da Silva s.n. (HAS103618); arroio Bom Jardim T1, 14-XI-2002, S.M. Alves da Silva s.n. (HAS103665); arroio Bom Jardim T1, 1-XII-2003, V.R. Werner s.n. (HAS104543); arroio Bom Jardim T1, 12-IV-2004, G.R. Cunha s.n. (HAS104783); arroio Bom Jardim
T3, 9-VIII-2004, G.R. Cunha s.n. (HAS106101); arroio Bom Jardim T2, 6-XII-2004, S.M. Alves da Silva s.n. (HAS106390); arroio Bom Jardim T3, 11-I-2005, G.R. Cunha s.n. (HAS 106408); arroio Bom Jardim T2, 14-III-2005, S.M. Alves da Silva s.n. (HAS106446); arroio Bom Jardim T3, 14-III-2005, S.M. Alves da Silva s.n. (HAS106449); arroio Bom Jardim T3, 12-V-2005, G.R. Cunha s.n. (HAS106565); arroio Bom Jardim T3, 9-I-2006, S.M. Alves da Silva s.n. (HAS106773); arroio Bom Jardim T3, 8-II-2006, S.M. Alves da Silva s.n. (HAS106786).

Distribuição no Brasil: Norte (Amazonas), Sul (Rio Grande do Sul, Santa Catarina).

Esta espécie caracteriza-se por apresentar um colarinho baixo e largo com ca. $1 \mu \mathrm{m}$ alt. e $5 \mu \mathrm{m}$ larg. No arroio ocorreu em temperatura da água de $11,7-26,3{ }^{\circ} \mathrm{C}$, temperatura do ar de $12,0-30,0^{\circ} \mathrm{C}, \mathrm{pH}$ de $6,0-7,1$, condutividade de $13,2-380 \mu \mathrm{S} \mathrm{cm}^{-1}$, demanda bioquímica de oxigênio de $0,7-3,5 \mathrm{mg} \mathrm{L}^{-1}$, matéria orgânica de 5,8-10,2 $\mathrm{mg} \mathrm{L}^{-1}$, oxigênio dissolvido de 2,8-8,9 $\mathrm{mg} \mathrm{L}^{-1}$, sílica de 10,0-19,2 $\mathrm{mg} \mathrm{L}^{-1} \mathrm{e}$ ortofosfato de 10,0-550,0 $\mu \mathrm{g} \mathrm{L}^{-1}$. Pode ser considerada uma espécie euritérmica por suportar amplitude de temperatura superior a $10^{\circ} \mathrm{C}$.

Strombomonas brevicaudata Conf. \& Joo, Cryptogamie: Algologie 15: 274, figs. 67 a,b. 1994. Figura 3

Lórica elíptica, 21-22 $\mu \mathrm{m}$ compr., 12-14 $\mu \mathrm{m}$ larg., $\mathrm{Rc} / 1=1,5-1,6$; pólo anterior terminando em colarinho reto, curto, com bordo levemente irregular, $3 \mu \mathrm{m}$ alt., $6 \mu \mathrm{m}$ larg.; pólo posterior termina em processo caudal curto, 1,5-2 $\mu \mathrm{m}$ compr.; parede hialina, com contorno levemente irregular.

Material examinado: BRASIL. Rio GRANDE Do Sul: Triunfo, arroio Bom Jardim T1, 14-XI-2002, S.M. Alves da Silva s.n. (HAS103665).

Distribuição no Brasil: Norte (Amazonas).

Primeiro registro para o Estado do Rio Grande do sul e segundo para o Brasil. Anteriormente foi registrada no lago Camaleão, no Estado do Amazonas por Conforti (1994). Atualmente sua distribuição é restrita a América do Norte e América do Sul. Essa espécie ocorreu em temperatura da água de $21,6{ }^{\circ} \mathrm{C}$, temperatura do ar de $19^{\circ} \mathrm{C}, \mathrm{pH}$ de 6,8 , condutividade de $13,2 \mu \mathrm{S} \mathrm{cm}^{-1}$, demanda bioquímica de oxigênio de 2,0 $\mathrm{mg} \mathrm{L}^{-1}$, matéria orgânica de $7,8 \mathrm{mg} \mathrm{L}^{-1}$, oxigênio dissolvido de $6,1 \mathrm{mg} \mathrm{L}^{-1}$, sílica de $7,5 \mathrm{mg} \mathrm{L}^{-1} \mathrm{e}$ ortofosfato de $40,0 \mu \mathrm{g} \mathrm{L}^{-1}$. 

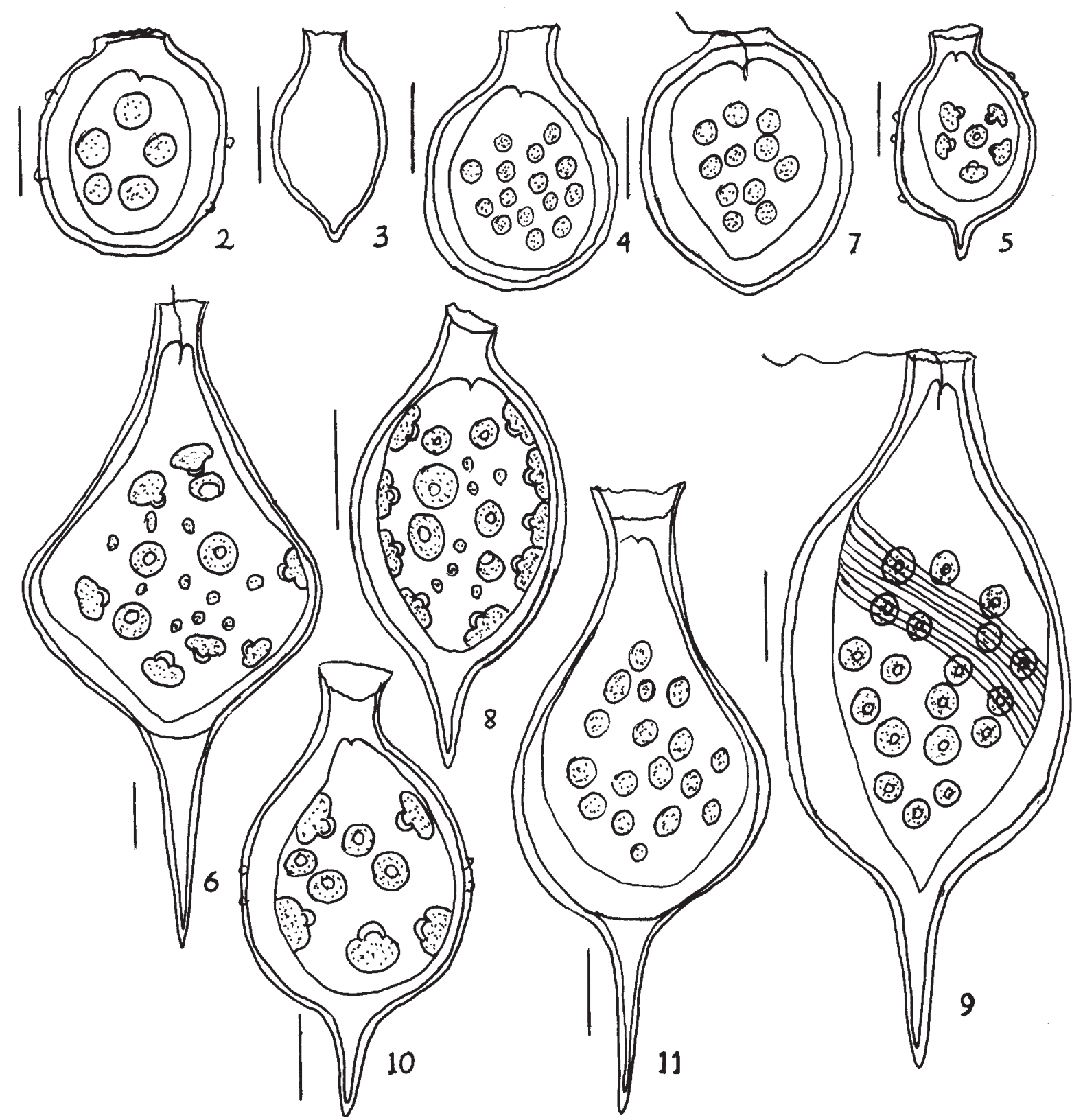

Figuras 2-11. Espécies de Strombomonas do arroio Bom Jardim, RS, Brasil. 2. Strombomonas borystheniensis (Roll) Pop. 3. S. brevicaudata Conf. \& Joo. 4. S. conspersa (Pascher) Tell \& Conf. 5. S. deflandrei (Roll) Defl. 6. S.ensifera (Daday) Defl. 7. S. eurystoma (Stein) f. incurva (Buz.) Pop. 8. S. fluviatilis var. fluviatilis (Lemm.) Defl. 9. S. fluviatilis (Lemm.) Defl. var. levis (Lemm.) Skv. 10-11. S. gibberosa var. gibberosa (Playf.) Defl. Escala $=10 \mu \mathrm{m}$.

Figures 2-11. Species of Strombomonas from Bom Jardim Stream, RS, Brazil. 2. Strombomonas borystheniensis (Roll) Pop. 3. S. brevicaudata Conf. \& Joo. 4. S. conspersa (Pascher) Tell \& Conf. 5. S. deflandrei (Roll) Defl. 6. S. ensifera (Daday) Defl. 7. S. eurystoma (Stein) f. incurva (Buz.) Pop. 8. S. fluviatilis var. fluviatilis (Lemm.) Defl. 9. S. fluviatilis (Lemm.) Defl. var. levis (Lemm.) Skv. 10-11. S. gibberosa var. gibberosa (Playf.) Defl. Scale $=10 \mu \mathrm{m}$. 
Strombomonas conspersa (Pascher) Tell \& Conf., Nova Hedwigia 40:124. 1984.

\section{Figura 4}

Lórica amplamente elíptica a arredondada, 28,5-30 $\mu \mathrm{m}$ compr., 20-24 $\mu \mathrm{m}$ larg.; Rc/1 = 1,3-1,4.

Material examinado: BRASIL. Rio GRANDE do SuL: Triunfo, arroio Bom Jardim T3, 6-II-2002, M.L. Nunes s.n. (HAS103195); arroio Bom Jardim T1, 6-II-2002, M.L. Nunes s.n. (HAS103202); arroio Bom Jardim T1, 2-IX-2002, S.M. Alves da Silva s.n. (HAS103615); arroio Bom Jardim T1, 14-XI-2002, S.M. Alves da Silva s.n. (HAS103665); arroio Bom Jardim T3, 8-II-2006, S.M. Alves da Silva s.n. (HAS106786).

Distribuição geográfica no Brasil: Sul (Rio Grande do Sul).

Célula semelhante a $S$. scabra var. scabra podendo ser distinguida pelo pólo anterior que em $S$. conspersa é mais longo, com colarinho terminando levemente oblíquo, 5-6 $\mu \mathrm{m}$ alt. 4-6 $\mu \mathrm{m}$ larg., enquanto em $S$. scabra o colarinho varia de 1-2,5 $\mu$ m alt. a 5-6 $\mu \mathrm{m}$ larg. Strombomonas conspersa ocorreu em temperatura da água de $16,2-26,3{ }^{\circ} \mathrm{C}$, temperatura do ar de $19,0-30,0^{\circ} \mathrm{C}$, pH de $6,8-6,9$, condutividade de $13,2-143,8 \mu \mathrm{S} \mathrm{cm}^{-1}$, demanda bioquímica de oxigênio de 2,0-2,6 $\mathrm{mg} \mathrm{L}^{-1}$, matéria orgânica de 5,8-10,2 $\mathrm{mg} \mathrm{L}^{-1}$, oxigênio dissolvido de 4,9-8,5 $\mathrm{mg} \mathrm{L}^{-1}$, sílica de 7,5-15,3 $\mathrm{mg} \mathrm{L}^{-1}$ e ortofosfato de 40,0-640,0 $\mu \mathrm{g} \mathrm{L}^{-1}$. Pode ser considerada uma espécie euritérmica por suportar amplitude de temperatura superior a $10^{\circ} \mathrm{C}$.

Strombomonas deflandrei (Roll) Defl., Arch.

Protistenk. 69(3): 575, fig. 37. 1930.

\section{Figura 5}

Lórica ovada, 30-33 $\mu \mathrm{m}$ compr., 19-20 $\mu \mathrm{m}$ larg.; $\mathrm{Rc} / 1=1,6-1,65$.

Material examinado: BRASIL. Rio GRANDE do Sul: Triunfo, arroio Bom Jardim T3, 6-II-2002, M.L. Nunes s.n. (HAS103195); arroio Bom Jardim T1, 6-II-2002, M.L. Nunes s.n. (HAS103202); arroio Bom Jardim T3, 2-XII-2002, S.M. Alves da Silva s.n. (HAS103696); arroio Bom Jardim T2, 6-XII-2004, S.M. Alves da Silva s.n. (HAS106390); arroio Bom Jardim T2, 11-I-2005, G.R. Cunha s.n. (HAS106405).

Distribuição geográfica no Brasil: Norte (Amazonas), Sul (Paraná, Rio Grande do Sul).

Essa espécie ocorreu em temperatura da água de $21,2-24,6{ }^{\circ} \mathrm{C}$, temperatura do ar de $21,7-30,0{ }^{\circ} \mathrm{C}$,
pH de 6,4-6,9, condutividade de 44,3-143,8 $\mu \mathrm{S} \mathrm{cm}^{-1}$, demanda bioquímica de oxigênio de 1,0-5,0 $\mathrm{mg} \mathrm{L}^{-1}$, matéria orgânica de $5,7-9,4 \mathrm{mg} \mathrm{L}^{-1}$, oxigênio dissolvido de 3,4-6,0 $\mathrm{mg} \mathrm{L}^{-1}$, sílica de 7,6-10,6 $\mathrm{mg} \mathrm{L}^{-1}$ e ortofosfato de $20,0-240,0 \mu \mathrm{g} \mathrm{L}^{-1}$.

Strombomonas ensifera (Daday) Defl., Arch. Protistenk. 69(3): 601, fig. 111-113, 115.1930.

Figura 6

Lórica rombóide, 93-103 $\mu \mathrm{m}$ compr., 41,5-44 $\mu \mathrm{m}$ larg., $\mathrm{Rc} / 1=2,2-2,3$.

Material examinado: BRASIL. Rio GRANDE DO Sul: Triunfo, arroio Bom Jardim T2, 25-III-2002, S.M. Alves da Silva s.n. (HAS103250); arroio Bom Jardim T3, 10-III-2003, S.M. Alves da Silva s.n. (HAS103952); arroio Bom Jardim T2, 14-III-2005, S.M. Alves da Silva s.n. (HAS106446).

Distribuição no Brasil: Norte (Amazonas), Nordeste (Maranhão), Centro-Oeste (Goiás, Mato Grosso), Sudeste (Rio de Janeiro), Sul (Paraná, Rio Grande do Sul).

Os indivíduos observados apresentaram haplopirenóides. Deflandre (1930) deu dimensões para essa espécie de 120-134 $\mu \mathrm{m}$ compr. e 38-55 $\mu \mathrm{m}$ larg. Nossos exemplares apresentam menor comprimento, entretanto as demais características conferem com as descritas para a espécie. Essa espécie ocorreu em temperatura da água de $24,5-29,9{ }^{\circ} \mathrm{C}$, temperatura do ar de $24,3-27,8^{\circ} \mathrm{C}, \mathrm{pH}$ de 6,9 , condutividade de $7,5-19,2 \mu \mathrm{S} \mathrm{cm}^{-1}$, demanda bioquímica de oxigênio de 1,7-2,8 $\mathrm{mg} \mathrm{L}^{-1}$, matéria orgânica de 8,0-9,2 $\mathrm{mg} \mathrm{L}^{-1}$, oxigênio dissolvido de 4,2-5,6 $\mathrm{mg} \mathrm{L}^{-1}$, sílica de $10,0-19,2 \mathrm{mg} \mathrm{L}^{-1}$ e ortofosfato de $70-150 \mu \mathrm{g} \mathrm{L}^{-1}$.

Strombomonas eurystoma (Stein) f. incurva (Buz.)

Pop., Flora plant. Cryptog. 3(1): 208, pl. 20, fig. 14, 16-21. 1966.

Figura 7

Lórica amplamente elíptica, 29-32 $\mu \mathrm{m}$ compr., 23-27,5 $\mu \mathrm{m}$ larg.; Rc/1 = 1,0-1,3; pólo anterior termina em colarinho curto e amplo, 1-2 $\mu \mathrm{m}$ alt.; 6-8 $\mu \mathrm{m}$ larg.; vista apical circular; pólo posterior levemente acuminado; parede hialina a amarelada, com aglutinações do meio; cloroplastos discóides, 2,8-3,5 $\mu \mathrm{m}$ diâm.; flagelo ca. de 1/3 do comprimento da lórica.

Material examinado: BRASIL. Rio GRANDE Do Sul: Triunfo, arroio Bom Jardim T3, 6-II-2002, M.L. Nunes s.n. (HAS103195); arroio Bom Jardim T1, 
6-II-2002, M.L. Nunes s.n. (HAS103202); arroio Bom Jardim T1, 14-XI-2002, S.M. Alves da Silva s.n. (HAS103665); arroio Bom Jardim T2, 9-I-2006, S.M. Alves da Silva s.n. (HAS106770).

Distribuição no Brasil: Primeira citação para o país.

Difere da espécie típica pelo pólo posterior acuminado. Brosnan et al. (2005) citaram que o pólo posterior acuminado depende dos movimentos celulares durante o seu deslocamento. Com base nesta afirmação, Conforti (2009) mencionou que a forma incurva poderia ser um morfotipo da espécie, com o que se discorda, visto que foram observados exemplares em quatro diferentes amostras e todos apresentaram o pólo posterior acuminado. Segundo Wolowski \& Walne (2007), essa forma ocorre em lagoas e rios com fluxo lento. No arroio Bom Jardim também foram encontradas em meses com fluxo lento. Essa forma ocorreu em temperatura da água que oscilou de $21,6-26,3{ }^{\circ} \mathrm{C}$, temperatura do ar de $19,0-30,0^{\circ} \mathrm{C}, \mathrm{pH}$ de $6,8-6,9$, condutividade de $13,2-143,8 \mu \mathrm{S} \mathrm{cm}^{-1}$, demanda bioquímica de oxigênio de 1,4-2,0 $\mathrm{mg} \mathrm{L}^{-1}$, matéria orgânica de 5,8-7,8 $\mathrm{mg} \mathrm{L}^{-1}$, oxigênio dissolvido de $4,1-6,1 \mathrm{mg} \mathrm{L}^{-1}$, sílica de 7,5-15,3 $\mathrm{mg} \mathrm{L}^{-1}$ e ortofosfato de 40,0-50,0 $\mu \mathrm{g} \mathrm{L}^{-1}$.

Strombomonas fluviatilis var. fluviatilis (Lemm.)

Defl., Arch. Protistenk. 69(3): 580, figs. 52-53. 1930.

Figura 8

Lórica elíptica, 27-40 $\mu \mathrm{m}$ compr., 15-22 $\mu \mathrm{m}$ larg., $\mathrm{Rc} / 1=1,8$.

Material examinado: BRASIL. Rio GRANDE Do Sul: Triunfo, arroio Bom Jardim T1, 6-II-2002, M.L. Nunes s.n. (HAS103202); arroio Bom Jardim T1, 14-XI-2002, S.M. Alves da Silva s.n. (HAS103665); arroio Bom Jardim T2, 6-I-2003, G.R. Cunha s.n. (HAS103833); arroio Bom Jardim T3, 1-XII-2003, V.R. Werner s.n. (HAS 104549); arroio Bom Jardim T2, 5-I-2004, S.M. Alves da Silva s.n. (HAS104564); arroio Bom Jardim T2, 14-III-2005, S.M. Alves da Silva s.n. (HAS106446); arroio Bom Jardim T3, 8-II-2006, S.M. Alves da Silva s.n. (HAS106786).

Distribuição no Brasil: Norte (Amazonas, Rondônia), Nordeste (Maranhão), Centro-Oeste (Goiás), Sudeste (Rio de Janeiro, São Paulo), Sul (Paraná, Santa Catarina, Rio Grande do Sul).

Os indivíduos observados apresentaram haplopirenóides. Essa variedade ocorreu em temperatura da água de $24,5-29,9{ }^{\circ} \mathrm{C}$, temperatura do ar de $19-30{ }^{\circ} \mathrm{C}$, pH de 6,5-7,1, condutividade de 13,2-670,0 $\mu \mathrm{S} \mathrm{cm}^{-1}$, demanda bioquímica de oxigênio

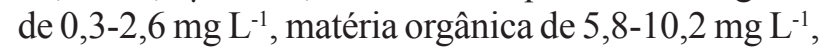
oxigênio dissolvido de $4,26,7 \mathrm{mg} \mathrm{L}^{-1}$, sílica de 7,5-15,3 $\mathrm{mg} \mathrm{L}^{-1}$ e ortofosfato de 10,0-380,0 $\mu \mathrm{g} \mathrm{L}^{-1}$. Pode ser considerada uma espécie euritérmica por suportar amplitude de temperatura superior a $10^{\circ} \mathrm{C}$.

Strombomonas fluviatilis (Lemm.) Defl. var. levis (Lemm.) Skv., Aus der Biol. Sungari Station zur Harbin 1(2): 78, pl. 6, fig. 33. 1925.

Figura 9

Lórica elíptica 68-75 $\mu \mathrm{m}$ compr., 30-35 $\mu \mathrm{m}$ larg., $\mathrm{Rc} / 1=2,1-2,3$; haplopirenóides presentes.

Material examinado: BRASIL. Rio GRANDE Do SuL: Triunfo, arroio Bom Jardim T1, 6-II-2002, M.L. Nunes s.n. (HAS103202); arroio Bom Jardim T3, 4-II-2003, G.R. Cunha s.n. (HAS103862); arroio Bom Jardim T3, 10-III-2003, S.M. Alves da Silva s.n. (HAS103952); arroio Bom Jardim T2, 9-I-2006, S.M. Alves da Silva s.n. (HAS106770).

Distribuição no Brasil: Nordeste (Paraíba), Sul (Rio Grande do Sul, Santa Catarina).

Essa variedade ocorreu em temperatura da água de $24,6-26,3{ }^{\circ} \mathrm{C}$, temperatura do ar de $27,8-30,0{ }^{\circ} \mathrm{C}$, $\mathrm{pH}$ de 6,7-6,9, condutividade de 82,8-484,0 $\mu \mathrm{S} \mathrm{cm}^{-1}$, demanda bioquímica de oxigênio de $1,4-2,8 \mathrm{mg} \mathrm{L}^{-1}$, matéria orgânica de $5,8-8,5 \mathrm{mg} \mathrm{L}^{-1}$, oxigênio dissolvido de 4,5-7,5 $\mathrm{mg} \mathrm{L}^{-1}$, sílica de $15,5-19,2 \mathrm{mg} \mathrm{L}^{-1}$ e ortofosfato de 50,0-590,0 $\mu \mathrm{g} \mathrm{L}^{-1}$.

Strombomonas gibberosa var. gibberosa (Playf.) Defl., Arch. Protistenk., 69(3): 595, fig. 97-101, 103.1930

Figuras 10-11

Lórica rombóide, região mediana a posterior alargada, 49-74 $\mu \mathrm{m}$ compr., 20-31 $\mu \mathrm{m}$ larg., $\mathrm{Rc} / 1=1,8-2,4$; processo caudal de 12-17 $\mu \mathrm{m}$ compr.

Material examinado: BRASIL. Rio Grande do Sul: Triunfo, arroio Bom Jardim T3, 6-II-2002, M.L. Nunes s.n. (HAS103195); arroio Bom Jardim T1, 6-II-2002, M.L. Nunes s.n. (HAS103202); arroio Bom Jardim T2, 6-II-2002, M.L. Nunes s.n. (HAS103188); arroio Bom Jardim T3, 10-III-2003, S.M. Alves da Silva s.n. (HAS103952); arroio Bom Jardim T3, 11-I-2005, G.R. Cunha s.n.(HAS106408); arroio Bom Jardim T2, 9-I-2006, S.M. Alves da Silva s.n. (HAS106770); arroio Bom Jardim T3, 8-II-2006, S.M. Alves da Silva s.n. (HAS106786). 
Distribuição no Brasil: Norte (Amazonas), Sudeste (Rio de Janeiro), Sul (Paraná, Rio Grande do Sul, Santa Catarina).

A maioria dos indivíduos apresentou dimensões entre 50-60 $\mu \mathrm{m}$ compr. e 20-26 $\mu \mathrm{m}$ larg., mas alguns poucos indivíduos apresentaram dimensões de 71-74 $\mu \mathrm{m}$ compr., próximas às dimensões dadas por Conforti (1993) para var. major (75-77 $\mu \mathrm{m}$ compr.). Como foram encontrados poucos indivíduos maiores, optou-se por identificá-los como a variedade típica da espécie. Essa variedade ocorreu em temperatura da água de $22,1-26,2^{\circ} \mathrm{C}$, temperatura do ar de $25,8-30^{\circ} \mathrm{C}$, $\mathrm{pH}$ de $6,5-6,9$, condutividade de $66,8-670,0 \mu \mathrm{S} \mathrm{cm}^{-1}$, demanda bioquímica de oxigênio de 1,4-3,5 $\mathrm{mg} \mathrm{L}^{-1}$, matéria orgânica de $5,8-10,2 \mathrm{mg} \mathrm{L}^{-1}$, oxigênio dissolvido de 2,8-5,8 $\mathrm{mg} \mathrm{L}^{-1}$, sílica de 15,3-19,2 $\mathrm{mg} \mathrm{L}^{-1}$ e ortofosfato de $50,0-550,0 \mathrm{mg} \mathrm{L}^{-1}$.

Strombomonas girardiana (Playf.) Defl. var. girardiana, Arch. Protistenk. 69(3): 573, fig. 33-35. 1930.

Figuras 12-14

Lórica subhexagonal, 37-52 $\mu \mathrm{m}$ comprimento, 20-27 $\mu \mathrm{m}$ larg., $\mathrm{Rc} / 1=1,7-2,0$.

Material examinado: BRASIL. Rio Grande do Sul: Triunfo, arroio Bom Jardim T1, 6-II-2002, M.L. Nunes s.n. (HAS103202); arroio Bom Jardim T3, 22-IV-2002, S.M. Alves da Silva s.n. (HAS103397); arroio Bom Jardim T2, 22-IV-2002, S.M. Alves da Silva s.n. (HAS103394); arroio Bom Jardim T1, 2-IX-2002, S.M. Alves da Silva s.n. (HAS103615); arroio Bom Jardim T1, 7-X-2002, G.R. Cunha s.n. (HAS103640); arroio Bom Jardim T1, 14-XI-2002, S.M. Alves da Silva s.n. (HAS103665); arroio Bom Jardim T1, 2-XII-2002, S.M. Alves da Silva s.n. (HAS 103690); arroio Bom Jardim T2, 2-XII-2002, S.M. Alves da Silva s.n. (HAS 103693); arroio Bom Jardim T2, 6-I-2003, G.R. Cunha s.n. (HAS103833); arroio Bom Jardim T3, 4-II-2003, G.R. Cunha s.n. (HAS103862); arroio Bom Jardim T2, 7-IV-2003, S.M. Alves da Silva s.n. (HAS103996), arroio Bom Jardim T3, 3-XI-2005, S.M. Alves da Silva s.n. (HAS104528); arroio Bom Jardim T3, 1-XII-2003, V.R. Werner s.n. (HAS 104549); arroio Bom Jardim T3, 5-I-2004, S.M. Alves da Silva s.n. (HAS104567); arroio Bom Jardim T1, 12-IV-2004, G.R. Cunha s.n. (HAS104783); arroio Bom Jardim T3, 11-V-2004, S.M. Alves da Silva s.n. (HAS104810); arroio Bom Jardim T3, 9-VIII-2004, G.R. Cunha s.n. (HAS106101); arroio Bom Jardim T2, 6-XII-2004, S.M. Alves da Silva s.n.
(HAS106390); arroio Bom Jardim T3, 11-I-2005, G.R. Cunha s.n. (HAS106408), arroio Bom Jardim T3, 14-III-2005, S.M. Alves da Silva s.n. (HAS106449); arroio Bom Jardim T1, 12-V-2005 G.R. Cunha s.n. (HAS106559); arroio Bom Jardim T2, 9-I-2006, S.M. Alves da Silva s.n. (HAS106770).

Distribuição no Brasil: Norte (Amazonas), Sudeste (Rio de Janeiro), Sul (Paraná, Rio Grande do Sul, Santa Catarina).

Foram observados indivíduos sem pirenóides e alguns com haplopirenóides. Essa variedade ocorreu em temperatura da água de $16,2-26,3^{\circ} \mathrm{C}$, temperatura do ar de $13,6-30^{\circ} \mathrm{C}$, pH de 6,0-7,1, condutividade de $13,2-260,0 \mu \mathrm{S} \mathrm{cm}^{-1}$, demanda bioquímica de oxigênio de 0,3-3,5 mg L-1, matéria orgânica de 0,3-4,2 $\mathrm{mg} \mathrm{L}^{-1}$, oxigênio dissolvido de $2,8-8,7 \mathrm{mg} \mathrm{L}^{-1}$, sílica de 4,5-19,2 $\mathrm{mg} \mathrm{L}^{-1}$ e ortofosfato de 10,0-640,0 $\mu \mathrm{g} \mathrm{L}^{-1}$. Pode ser considerada uma espécie euritérmica por suportar amplitude de temperatura superior a $10^{\circ} \mathrm{C}$.

Strombomonas girardiana (Playf.) Defl. var. triondulata Tell \& Zaloc., Nova Hedwigia 41:373, pl 13, fig. 4 a, b. 1985.

Figura 15

Lórica subhexagonal, lados com duas reentrâncias nas margens laterais, 45-50,5 $\mu \mathrm{m}$ compr., 23-27 $\mu \mathrm{m}$ larg., $\mathrm{Rc} / 1=1,6-2,2$.

Material examinado: BRASIL. Rio GRANDE Do Sul: Triunfo, arroio Bom Jardim T3, 3-XI-2005, S.M. Alves da Silva s.n. (HAS104528).

Distribuição no Brasil: Sudeste (Rio de Janeiro), Sul (Paraná, Rio Grande do Sul).

Os indivíduos observados apresentaram haplopirenóides. Esta variedade ocorreu em temperatura da água de $18,1^{\circ} \mathrm{C}$, temperatura do ar de $20,4{ }^{\circ} \mathrm{C}, \mathrm{pH}$ de 6,9 , condutividade de $122,2 \mu \mathrm{S} \mathrm{cm}^{-1}$, demanda bioquímica de oxigênio de $2,0 \mathrm{mg} \mathrm{L}^{-1}$, matéria orgânica de $8,0 \mathrm{mg} \mathrm{L}^{-1}$, oxigênio dissolvido de $6,9 \mathrm{mg} \mathrm{L}^{-1}$, sílica de $13,2 \mathrm{mg} \mathrm{L}^{-1}$ e ortofosfato de $280 \mathrm{mg} \mathrm{L}^{-1}$.

Strombomonas maxima (Skv.) Defl., Arch. Protistenk. 69(3): 583-584, fig. 61-65. 1930.

Figura 16

Lórica ovada, 74-98 $\mu \mathrm{m}$ compr., 33,5-41 $\mu \mathrm{m}$ larg., $\mathrm{Rc} / 1=2,2-2,6$.

Material examinado: BRASIL. Rio Grande Do Sul: Triunfo, arroio Bom Jardim T1, 6-II-2002, M.L. 


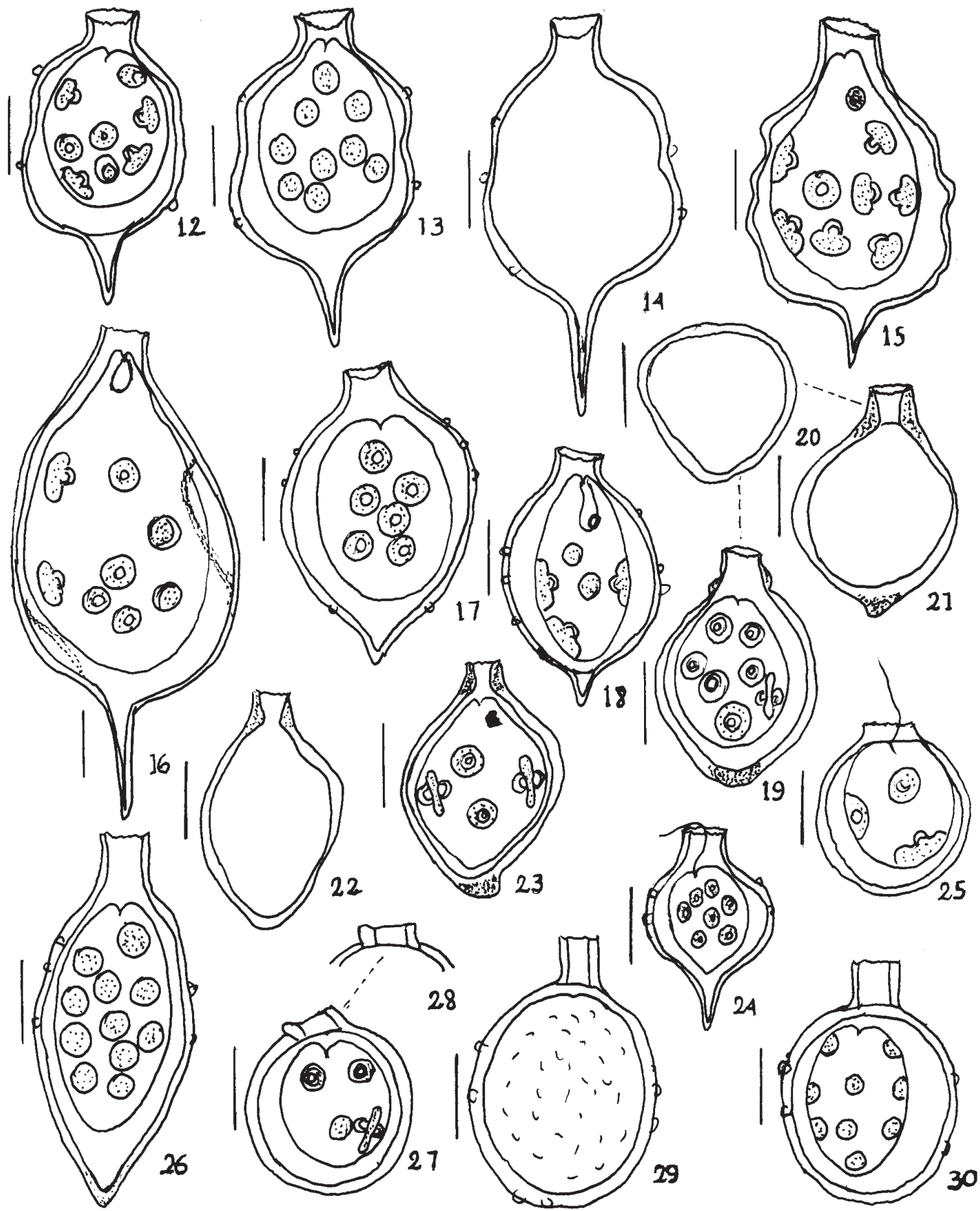

Figuras 12-30. Espécies de Strombomonas do arroio Bom Jardim, RS, Brasil. 12-14. S. girardiana (Playf.) Defl . var. girardiana. 15. S. girardiana (Playf.) Defl. var. triondulata Tell \& Zaloc. 16. S. maxima (Skv.) Defl. 17-18. S. ovalis (Playf.) Defl. 19-23. S. recurvata Conf. \& Joo, 20. vista apical, 22. vista lateral. 24. S. rotunda (Playf.) Defl., 25. S. scabra (Playf.) Tell \& Conf. var. Scabra. 26. S. scabra (Playf.) Tell \& Conf. var. intermedia (Yacubson) Tell \& Conf. 27-28. S. scabra (Playf.) Tell \& Conf. var. labiata (Hub.-Pest.) Tell \& Conf., 28. detalhe do colarinho. 29-30. S. scabra (Playf.) Tell \& Conf. var. longicollis (Playf.) Tell \& Conf. Escala $=10 \mu \mathrm{m}$.

Figures 12-30. Species of Strombomonas from the Bom Jardim stream, RS, Brazil. 12-14. S. girardiana (Playf.) Defl . var. girardiana. 15. S. girardiana (Playf.) Defl. var. triondulata Tell \& Zaloc. 16. S. maxima (Skv.) Defl. 17-18. S. ovalis (Playf.) Defl. 19-23. S. recurvata Conf. \& Joo, 20. apical view. 22. lateral view. 24. S. rotunda (Playf.) Defl., 25. S. scabra (Playf.) Tell \& Conf. var. Scabra. 26. S. scabra (Playf.) Tell \& Conf. var. intermedia (Yacubson) Tell \& Conf. 27-28. S. scabra (Playf.) Tell \& Conf. var. labiata (Hub.-Pest.) Tell \& Conf., 28. detail of collar. 29-30. S. scabra (Playf.) Tell \& Conf. var. longicollis (Playf.) Tell \& Conf. Scale $=10 \mu \mathrm{m}$. 
Nunes s.n. (HAS103202); arroio Bom Jardim T3, 2-XII-2002, S.M. Alves da Silva s.n. (HAS103696); arroio Bom Jardim T3, 6-I-2003, G.R. Cunha s.n. (HAS103836); arroio Bom Jardim T1, 7-IV-2003, S.M. Alves da Silva s.n. (HAS103993); arroio Bom Jardim T3, 7-IV-2003, S.M. Alves da Silva s.n. (HAS 103999); arroio Bom Jardim T3, 15-II-2005, S.M. Alves da Silva s.n. (HAS106429); arroio Bom Jardim T2, 14-III-2005, S.M. Alves da Silva s.n. (HAS106446); arroio Bom Jardim T3, 8-XI-2005, S.M. Alves da Silva s.n. (HAS106721).

Distribuição no Brasil: Norte (Pará, Acre), Centro-Oeste (Mato Grosso), Sudeste (Rio de Janeiro), Sul (Paraná, Rio Grande do Sul).

Os indivíduos observados apresentaram haplopirenóides. Esta espécie ocorreu em temperatura da água de $18,7-25,3{ }^{\circ} \mathrm{C}$, temperatura do ar de 20,0-30,0 ${ }^{\circ} \mathrm{C}, \mathrm{pH}$ de 5,6-6,9, condutividade de $53,4-517 \mu \mathrm{S} \mathrm{cm}^{-1}$, demanda bioquímica de oxigênio de 0,5-4,4 mg L-1, matéria orgânica de 5,8-9,5 $\mathrm{mg} \mathrm{L}^{-1}$, oxigênio dissolvido de $3,7-6,5 \mathrm{mg} \mathrm{L}^{-1}$, sílica de 7,6 - 20,6 $\mathrm{mg} \mathrm{L}^{-1}$ e ortofosfato de 70,0 - 940,0 $\mu \mathrm{g} \mathrm{L}^{-1}$.

Strombomnas ovalis (Playf.) Defl. Arch. Protistenk. 69(3): 577, fig. 42-43. 1930.

Figuras 17-18

Lórica ovada 31-35 $\mu \mathrm{m}$ compr., 19-23 $\mu \mathrm{m}$ larg., $\mathrm{Rc} / 1=1,6-1,8$.

Material examinado: BRASIL. Rio Grande do Sul: Triunfo, arroio Bom Jardim T2, 6-II-2002, M.L. Nunes s.n. (HAS103188); arroio Bom Jardim T1, 6-II-2002, M.L.Nunes s.n. (HAS103202); arroio Bom Jardim T3, 7-VI-2004, S.M. Alves da Silva s.n. (HAS 104827).

Distribuição geográfica: Norte (Amazonas, Rondônia), Sudeste (Rio de Janeiro), Sul (Paraná, Rio Grande do Sul).

Os indivíduos observados apresentaram haplopirenóides. Segundo Tell \& Conforti (1986), essa espécie é provavelmente cosmopolita, tendo ampla distribuição na Argentina em ambientes lênticos e lóticos, ricos em matéria orgânica, e sendo encontrada no Brasil também em lagoa rasa, lago e rio (Conforti 1993, Alves-da-Silva \& Torres 1994a, Train \& Rodrigues 2004). Essa espécie ocorreu em temperatura da água de $13,2-24,6{ }^{\circ} \mathrm{C}$, temperatura do ar de $16,2-30,0{ }^{\circ} \mathrm{C}, \mathrm{pH}$ de $6,5-6,7$, condutividade de $66,8-122,2 \mu \mathrm{S} \mathrm{cm}^{-1}$, demanda bioquímica de oxigênio de 1,3 $\mathrm{mg} \mathrm{L}^{-1}$, matéria orgânica de 5,8-9,6 mg L-1, oxigênio dissolvido de $5,8-7,1 \mathrm{mg} \mathrm{L}^{-1}$, sílica de $15,6 \mathrm{mg} \mathrm{L}^{-1}$ e ortofosfato de $290 \mu \mathrm{g} \mathrm{L}^{-1}$. Pode ser considerada uma espécie euritérmica por suportar amplitude de temperatura superior a $10^{\circ} \mathrm{C}$.

Strombomonas recurvata Conf. \& Joo, Cryptogamie. Algol. 15(4): 277, fig. 76 a-g. 1994.

Figuras 19-23

Lórica ovada, 26,5-30,2 $\mu \mathrm{m}$ compr., 18-20 $\mu \mathrm{m}$ larg., $\mathrm{Rc} / 1=1,4-1,5$; vista polar triangular; vista lateral de elíptica a triangular; pólo anterior termina em colarinho cilíndrico, com espessamento na sua base, ca. $5 \mu \mathrm{m}$ alt., 4,5-5 $\mu \mathrm{m}$ larg.; pólo posterior levemente acuminado em processo caudal mamilado irregular; parede espessa, castanho-avermelhada, cloroplastos com duplopirenóides.

Material examinado: BRASIL. Rio Grande Do Sul: Triunfo, arroio Bom Jardim T2, 6-II-2002, M.L. Nunes s.n. (HAS103188); arroio Bom Jardim T1, 6-II-2002, M.L. Nunes s.n. (HAS103202); arroio Bom Jardim T1, 2-IX-2002, S.M. Alves da Silva s.n. (HAS103615); arroio Bom Jardim T2, 2-IX-2002, S.M. Alves da Silva s.n. (HAS103618); arroio Bom Jardim T1, 1-XII-2003, V.R. Werner s.n. (HAS104543); arroio Bom Jardim T3, 1-XII-2003, V.R. Werner s.n. (HAS 104549).

Distribuição geográfica: primeira citação para o país.

Os indivíduos encontrados foram ligeiramente maiores que os registrados nos Estados Unidos por Conforti \& Joo (1994); e a vista apical observada foi triangular e não em forma de pêra como mostrado na figura 76e pelas autoras supra citadas. As demais características conferem com as descritas para a espécie. Strombomonas recurvata apresenta semelhança com S. scabra var. ovata podendo ser distinguidas pela vista apical, que em var. ovata é circular e em $S$. recurvata é triangular; e também pelo menor colarinho com espessamento na sua base e pela vista lateral levemente triangular. A espécie foi descrita em clima temperado em lagos meandricos de Indiana (USA). Presentemente foi registrada em clima subtropical, ampliando o conhecimento da sua distribuição.

No arroio Bom Jardim ocorreu em temperatura da água que oscilou de $11,7-26,3{ }^{\circ} \mathrm{C}$, temperatura do ar de $11,8-25,8^{\circ} \mathrm{C}, \mathrm{pH}$ de $6,5-6,8$, condutividade de $38,4-211 \mu \mathrm{S} \mathrm{cm}^{-1}$, demanda bioquímica de oxigênio de $0,7-2,1 \mathrm{mg} \mathrm{L}^{-1}$,matéria orgânica de 5,8-9,8 $\mathrm{mg} \mathrm{L}^{-1}$, 
oxigênio dissolvido de 5,8-8,9 $\mathrm{mg} \mathrm{L}^{-1}$, sílica de $6,1-12,3 \mathrm{mg} \mathrm{L}^{-1}$ e ortofosfato de 10-640 $\mu \mathrm{g} \mathrm{L}^{-1}$. Pode ser considerada uma espécie euritérmica por suportar amplitude de temperatura da água superior a $10^{\circ} \mathrm{C}$.

Strombomonas rotunda (Playf.) Defl., Arch. Protistenk. 69(3): 593, fig. 88, 1930.

\section{Figura 24}

Lórica ovada a transversalmente elíptica, 24,5-27 $\mu \mathrm{m}$ comprimento, 16-17 $\mu \mathrm{m}$ larg., $\mathrm{Rc} / 1=1,6-1,7$.

Material examinado: BRASIL. Rio GRANDE do Sul: Triunfo, arroio Bom Jardim T1, 14-XI-2002, S.M. Alves da Silva s.n. (HAS103665).

Distribuição no Brasil: Nordeste (Paraíba), Norte (Amazonas), Sul (Rio Grande do Sul, Santa Catarina), Sudeste (Rio de Janeiro, São Paulo).

Essa espécie ocorreu em temperatura da água de $21,6{ }^{\circ} \mathrm{C}$, temperatura do ar de $19,0{ }^{\circ} \mathrm{C}$, $\mathrm{pH}$ de 6,8 , condutividade de $13,2 \mu \mathrm{S} \mathrm{cm}^{-1}$, demanda bioquímica de oxigênio de $2,0 \mathrm{mg} \mathrm{L}^{-1}$, matéria orgânica de $7,8 \mathrm{mg} \mathrm{L}^{-1}$, oxigênio dissolvido de $6,1 \mathrm{mg} \mathrm{L}^{-1}$, sílica de $7,5 \mathrm{mg} \mathrm{L}^{-1}$ e ortofosfato de $40,0 \mu \mathrm{g} \mathrm{L}^{-1}$.

Strombomonas scabra (Playf.) Tell \& Conf. var. scabra, Nova Hedwigia 46(3-4): 545, pl. 1, fig. 1-6, pl. 6, fig. 1-3. 1988.

Figura 25

Lórica amplamente elíptica a globosa, 18-26 $\mu \mathrm{m}$ compr., 15-21,3 $\mu \mathrm{m}$ larg., Rc/1 = 1,2.

Material examinado: BRASIL. Rio GRANDE Do Sul: Triunfo, arroio Bom Jardim T3, 6-II-2002, M.L. Nunes s.n. (HAS103195); arroio Bom Jardim T1, 6-II-2002, M.L. Nunes s.n. (HAS103202); arroio Bom Jardim T3, 22-IV-2002, S.M. Alves da Silva s.n. (HAS103397); arroio Bom Jardim T2, 22-IV-2002, S.M. Alves da Silva s.n. (HAS103394); arroio Bom Jardim T1, 3-VII-2002, S.M. Alves da Silva s.n. (HAS 103457); arroio Bom Jardim T2, 3-VII-2002, S.M. Alves da Silva s.n. (HAS103459); arroio Bom Jardim T2, 5-VIII-2002, G.R. Cunha s.n. (HAS103524); arroio Bom Jardim T1, 2-IX-2002, S.M. Alves da Silva s.n. (HAS103615); arroio Bom Jardim T2, 2-IX-2002, S.M. Alves da Silva s.n. (HAS103618); arroio Bom Jardim T3, 2-IX-2002, S.M. Alves da Silva s.n. (HAS103621); arroio Bom Jardim T1, 7-X-2002, G.R. Cunha s.n. (HAS103640); arroio Bom Jardim T2, 7-X-2002, G.R. Cunha s.n. (HAS103643); arroio Bom Jardim T1, 14-XI-2002, S.M. Alves da Silva s.n. (HAS103665); arroio Bom Jardim T3, 2-XII-2002, S.M. Alves da Silva s.n.
(HAS103696); arroio Bom Jardim T1, 6-I-2003, G.R. Cunha s.n. (HAS103830); arroio Bom Jardim T1, 4-II-2003, G.R. Cunha s.n. (HAS103856); arroio Bom Jardim T3, 4-II-2003, G.R. Cunha s.n. (HAS103862); arroio Bom Jardim T2, 4-II-2003, G.R. Cunha s.n. (HAS103859); arroio Bom Jardim T2, 10-III-2003, S.M. Alves da Silva s.n. (HAS103949); arroio Bom Jardim T1, 7-IV-2003, S.M. Alves da Silva s.n. (HAS103993); arroio Bom Jardim T1, 5-V-2003, G.R. Cunha s.n. (HAS104014); arroio Bom Jardim T2, 5-V-2003, G.R. Cunha s.n. (HAS104017); arroio Bom Jardim T1, 2-VI-2003, S.M. Alves da Silva s.n. (HAS104039); arroio Bom Jardim T1, 3-XI-2003, S.M. Alves da Silva s.n.(HAS104522); arroio Bom Jardim T2, 3-XI-2003, S.M. Alves da Silva s.n. (HAS104525); arroio Bom Jardim T1, 1-XII-2003, V.R. Werner s.n. (HAS104543); arroio Bom Jardim T3, 1-XII-2003, V.R. Werner s.n. (HAS 104549); arroio Bom Jardim T1, 5-I-2004, S.M. Alves da Silva s.n. (HAS104561); arroio Bom Jardim T3, 13-II-2004, G.R. Cunha s.n. (HAS104850); arroio Bom Jardim T2, 9-VIII-2004, G.R. Cunha s.n. (HAS106098); arroio Bom Jardim T3, 9-VIII-2004, G.R. Cunha s.n. (HAS106101); arroio Bom Jardim T1, 13-IX-2004, S.M. Alves da Silva s.n. (HAS106116); arroio Bom Jardim T2, 13-IX-2004, S.M. Alves da Silva s.n. (HAS106119); arroio Bom Jardim T3, 13-IX-2004, S.M. Alves da Silva s.n. (HAS106122); arroio Bom Jardim T3, 7-X-2004, G.R. Cunha s.n. (HAS106143); arroio Bom Jardim T2, 6-XII-2004, S.M. Alves da Silva s.n. (HAS106390); arroio Bom Jardim T3, 6-XII-2004, S.M. Alves da Silva s.n. (HAS106393); arroio Bom Jardim T3, 14-III-2005, S.M. Alves da Silva s.n. (HAS106449); arroio Bom Jardim T2, 14-III-2005, S.M. Alves da Silva s.n. (HAS106446); arroio Bom Jardim T3, 8-XI-2005, S.M. Alves da Silva s.n. (HAS106721); arroio Bom Jardim T2, 8-II-2005, S.M. Alves da Silva s.n. (HAS106783).

Distribuição no Brasil: Centro-Oeste (Mato Grosso), Sudeste (Rio de Janeiro, São Paulo), Sul (Paraná, Rio Grande do Sul).

Alguns indivíduos apresentaram haplopirenóides e flagelo com metade do comprimento da lórica.

Essa variedade ocorreu em temperatura da água de $9,7-33,6{ }^{\circ} \mathrm{C}$, temperatura do ar de $11,8-30,0{ }^{\circ} \mathrm{C}$, $\mathrm{pH}$ de 6,5-7,0, condutividade de 13,2-418 $\mu \mathrm{S} \mathrm{cm}^{-1}$, demanda bioquímica de oxigênio de $0,5-3,4 \mathrm{mg} \mathrm{L}^{-1}$, matéria orgânica de $3,9-9,8 \mathrm{mg} \mathrm{L}^{-1}$, oxigênio dissolvido de 4,0-10,0 $\mathrm{mg} \mathrm{L}^{-1}$, sílica de 4,5-15,4 mg $\mathrm{L}^{-1}$ e ortofosfato de $10,0-950,0 \mu \mathrm{g} \mathrm{L}^{-1}$. Pode ser considerada uma espécie euritérmica por suportar amplitude de temperatura da água superior a $10^{\circ} \mathrm{C}$. 
Strombomonas scabra (Playf.) Tell \& Conf. var. intermedia (Yacubson) Tell \& Conf., Nova Hedwigia 46(3-4): 545, pl. 6, fig. 13-16. 1988.

Figura 26

Lórica elíptica, 41-45 $\mu \mathrm{m}$ compr., 18,5-21 $\mu \mathrm{m}$ larg., $\mathrm{Rc} / 1=2,1-2,2$.

Material examinado: BRASIL. Rio GRANDE do Sul: Triunfo, arroio Bom Jardim T2, 25-III-2003, S.M. Alves da Silva s.n. (HAS103250); arroio Bom Jardim T1, 2-IX-2002, S.M. Alves da Silva s.n. (HAS103615); arroio Bom Jardim T1, 4-II-2003, G.R. Cunha s.n. (HAS103856); arroio Bom Jardim T3, 10-III-2003, S.M. Alves da Silva s.n. (HAS103952); arroio Bom Jardim T3, 7-IV-2003, S.M. Alves da Silva s.n. (HAS 103999); arroio Bom Jardim T2, 3-XI-2003, S.M. Alves da Silva s.n. (HAS104525); arroio Bom Jardim T1, 1-XII-2003, V.R. Werner s.n. (HAS104543); arroio Bom Jardim T1, 7-X-2004, G.R. Cunha s.n. (HAS106137); arroio Bom Jardim T1, 8-XI-2004, S.M. Alves da Silva s.n. (HAS106158); arroio Bom Jardim T2, 14-III-2005, S.M. Alves da Silva s.n. (HAS106446); arroio Bom Jardim T3, 14-III-2005, S.M. Alves da Silva s.n. (HAS106449); arroio Bom Jardim T3, 12-V-2005, G.R. Cunha s.n. (HAS106565); arroio Bom Jardim T3, 8-II-2006, S.M. Alves da Silva s.n. (HAS106786).

Distribuição no Brasil: Norte (Amazonas), Sul (Rio Grande do Sul).

Difere da variedade típica pela forma, pelo colarinho de 5,5-6,5 $\mu \mathrm{m}$ alt., 4-5,5 $\mu \mathrm{m}$ larg. e processo caudal ca. $3 \mu \mathrm{m}$ compr. Foram observados alguns exemplares com haplopirenóides, como já registrados anteriormente por Alves-da-Silva \& Bridi (2004). Essa variedade ocorreu em temperatura da água de $16,2-33,6{ }^{\circ} \mathrm{C}$, temperatura do ar de $13,5-30,6{ }^{\circ} \mathrm{C}, \mathrm{pH}$ de $6,3-6,8$, condutividade de $47-670 \mu \mathrm{S} \mathrm{cm}^{-1}$, demanda bioquímica de oxigênio de $0,7-4,2 \mathrm{mg} \mathrm{L}^{-1}$, matéria orgânica de 5,2-10,2 $\mathrm{mg} \mathrm{L}^{-1}$, oxigênio dissolvido de 4,2-8,2 $\mathrm{mg} \mathrm{L}^{-1}$, sílica de 5,7-20,6 $\mathrm{mg} \mathrm{L}^{-1}$ e ortofosfato de 10,0-950,0 $\mu \mathrm{g} \mathrm{L}^{-1}$. Pode ser considerada uma espécie euritérmica por suportar amplitude de temperatura superior a $10^{\circ} \mathrm{C}$.

Strombomonas scabra (Playf.) Tell \& Conf. var. labiata (Hub.-Pest.) Tell \& Conf., Nova Hedwigia. 46(3-4): 545-546, pl. 6, fig. 4-5. 1988.

Figuras 27-28

Lórica ovada a elíptica, 21,5-22,0 $\mu \mathrm{m}$ compr., 16,0-19,0 $\mu \mathrm{m}$ larg., Rc/1 = 1,2-1,3.
Material examinado: BRASIL. Rio Grande do Sul: Triunfo, arroio Bom Jardim T1, 2-IX-2002, S.M. Alves da Silva s.n. (HAS103615).

Distribuição no Brasil: Norte (Amazonas), Sul (Rio Grande do Sul).

O pólo anterior termina em um colarinho bem diferenciado, ca. $4 \mu \mathrm{m}$ alt., 6,0-6,5 $\mu \mathrm{m}$ larg. Foi observada a presença de duplopirenóides, estrutura não registrada anteriormente, o que amplia a circunscrição dessa variedade, que ocorreu em temperatura da água de $16,2{ }^{\circ} \mathrm{C}$, temperatura do ar de $13,6{ }^{\circ} \mathrm{C}, \mathrm{pH}$ de 6,8 , condutividade de $38,4 \mu \mathrm{S} \mathrm{cm}^{-1}$, demanda bioquímica de oxigênio de $2,1 \mathrm{mg} \mathrm{L}^{-1}$, matéria orgânica de 7,5 $\mathrm{mg} \mathrm{L}^{-1}$, oxigênio dissolvido de $8,5 \mathrm{mg} \mathrm{L}^{-1}$, sílica de $12,3 \mathrm{mg} \mathrm{L}^{-1}$ e ortofosfato de $640 \mu \mathrm{g} \mathrm{L}^{-1}$.

Strombomonas scabra (Playf.) Tell \& Conf. var. longicollis (Playf.) Tell \& Conf., Nova Hedwigia 46(3-4): 546, pl. 2, fig. 4, pl. 7, fig. 1. 1988.

Figuras 29-30

Lórica ovada, 30,5-33 $\mu \mathrm{m}$ compr., 21,3-24 $\mu \mathrm{m}$ larg., $\mathrm{Rc} / 1=1,4-1,5$.

Material examinado: BRASIL. Rio GRANDE do Sul: Triunfo, arroio Bom Jardim T3, 3-VII-2002, S.M. Alves da Silva s.n. (HAS103458); arroio Bom Jardim T1, 2-IX-2002, S.M. Alves da Silva s.n. (HAS103615); arroio Bom Jardim T2, 2-IX-2002, S.M. Alves da Silva s.n. (HAS103618); arroio Bom Jardim T1, 14-XI-2002, S.M. Alves da Silva s.n. (HAS103665); arroio Bom Jardim T1, 2-XII-2002, S.M. Alves da Silva s.n. (HAS 103690); arroio Bom Jardim T1, 6-I-2003, G.R. Cunha s.n. (HAS103830); arroio Bom Jardim T3, 10-III-2003, S.M. Alves da Silva s.n. (HAS103952); arroio Bom Jardim T1, 1-XII-2003, V.R. Werner s.n. (HAS104543); arroio Bom Jardim T2, 1-XII-2003, V.R. Werner s.n. (HAS104546); arroio Bom Jardim T1, 5-I-2004, S.M. Alves da Silva s.n. (HAS104561); arroio Bom Jardim T2, 5-I-2004, S.M. Alves da Silva s.n. (HAS104564); arroio Bom Jardim T2, 7-X-2004, G.R. Cunha s.n. (HAS106140); arroio Bom Jardim T3, 7-X-2004, G.R. Cunha s.n. (HAS106143); arroio Bom Jardim T1, 8-XI-2004, S.M. Alves da Silva s.n. (HAS106158); arroio Bom Jardim T1, 11-IV-2005, S.M. Alves da Silva s.n. (HAS106546).

Distribuição no Brasil: Norte (Amazonas), CentroOeste (Mato Grosso), Sudeste (Rio de Janeiro), Sul (Rio Grande do Sul). 
Difere da variedade típica da espécie pela presença de colarinho alto, cilíndrico, reto ou levemente oblíquo, com bordo crenulado, 4,5-6,0 $\mu \mathrm{m}$ alt., 4,0-4,5 $\mu \mathrm{m}$ larg. Essa variedade ocorreu em temperatura da água de $11,7-33,6^{\circ} \mathrm{C}$, temperatura do ar de $11,8-28,4^{\circ} \mathrm{C}, \mathrm{pH}$ de $6,0-6,9$, condutividade de $13,2-489 \mu \mathrm{S} \mathrm{cm}^{-1}$, demanda bioquímica de oxigênio de $0,8-2,8 \mathrm{mg} \mathrm{L}^{-1}$, matéria orgânica de 3,9-10,7 $\mathrm{mg} \mathrm{L}^{-1}$, oxigênio dissolvido de 4,0-8,9 $\mathrm{mg} \mathrm{L}^{-1}$, sílica de 5,7-19,2 $\mathrm{mg} \mathrm{L}^{-1}$ e ortofosfato de 10,0-640,0 $\mu \mathrm{g} \mathrm{L}^{-1}$. Pode ser considerada uma espécie euritérmica por suportar amplitude de temperatura superior a $10^{\circ} \mathrm{C}$.

Strombomonas scabra (Playf.) Tell \& Conf. var. ovata (Playf.) Tell \& Conf. f. ovata (Defl.) Tell \& Conf., Nova Hedwigia 46(3-4): 546, pl. 2, fig. 5-6, pl.6, fig. 8-10. 1988.

Figuras 31-32

Lórica elíptica a ovada, 30-32 $\mu \mathrm{m}$ compr., 18,5-21,5 $\mu \mathrm{m}$ larg., $\mathrm{Rc} / \mathrm{l}=1,4-1,7$.

Material examinado: BRASIL. Rio GRANDE Do Sul: Triunfo, arroio Bom Jardim T1, 2-IX-2002, S.M. Alves da Silva s.n. (HAS103615); arroio Bom Jardim T2, 2-IX-2002, S.M. Alves da Silva s.n. (HAS103618); arroio Bom Jardim T1, 14-XI-2002, S.M. Alves da Silva s.n. (HAS103665); arroio Bom Jardim T1, 1-XI-2003, S.E. Salomoni s.n. (HAS104298); arroio Bom Jardim T1, 1-XII-2003, V.R. Werner s.n. (HAS 104543); arroio Bom Jardim T3, 8-II-2006, S.M. Alves da Silva s.n. (HAS106786).

Distribuição no Brasil: Sul (Rio Grande do Sul).

$\mathrm{O}$ pólo anterior atenua abruptamente terminando em colarinho alto, com bordo crenulado com ca. $4 \mu \mathrm{m}$ compr. e 3,5 $\mu \mathrm{m}$ larg.; haplopirenóides presentes. Difere da variedade típica da espécie por sua lórica elíptica a ovada e por apresentar o pólo posterior levemente atenuado em processo caudal com até $3 \mu \mathrm{m}$ de comprimento. Essa variedade ocorreu em temperatura da água de $11,7-26,3^{\circ} \mathrm{C}$, temperatura do ar de $11,8-28,4{ }^{\circ} \mathrm{C}$, pH de $6,2-6,8$, condutividade de $11,7-670,0 \mu \mathrm{S} \mathrm{cm}^{-1}$, demanda bioquímica de oxigênio de 0,7-2,6 $\mathrm{mg} \mathrm{L}^{-1}$, matéria orgânica de 7,4-15,3 $\mathrm{mg} \mathrm{L}^{-1}$, oxigênio dissolvido de 4,9-8,9 $\mathrm{mg} \mathrm{L}^{-1}$, ś́lica de 7,5-15,3 $\mathrm{mg} \mathrm{L}^{-1}$ e ortofosfato de 10,0-640,0 $\mu \mathrm{g} \mathrm{L}^{-1}$. Pode ser considerada uma espécie euritérmica por suportar amplitude de temperatura superior a $10^{\circ} \mathrm{C}$.

Strombomonas scabra (Playf.) Tell \& Conf. var. ovata (Playf.) Tell \& Conf. f. minor Tell \& Conf., Nova Hedwigia 46(3-4): 546, pl. 2, fig. 2-3, pl. 6, fig. 11-12. 1988.

Figuras 33-34
Lorica elíptica, alargada na região anterior, 20-21 $\mu \mathrm{m}$ compr., 13-15 $\mu \mathrm{m}$ larg., $\mathrm{Rc} / \mathrm{l}=1,3-1,5$.

Material examinado: BRASIL. Rio GRANDE do SUL: Triunfo, arroio Bom Jardim T1, 6-II-2002, M.L. Nunes s.n. (HAS103202); arroio Bom Jardim T2, 25-III-2002, S.M. Alves da Silva s.n. (HAS103250); arroio Bom Jardim T2, 5-VIII-2002, G.R. Cunha s.n. (HAS103524); arroio Bom Jardim T1, 2-IX-2002, S.M. Alves da Silva s.n. (HAS103615); arroio Bom Jardim T3, 2-IX-2002, S.M. Alves da Silva s.n. (HAS 103621); arroio Bom Jardim T2, 2-IX-2002, S.M. Alves da Silva s.n. (HAS103618); arroio Bom Jardim T2, 7-X-2002, G.R. Cunha s.n. (HAS103643); arroio Bom Jardim T1, 1-VII-2003, S.M. Alves da Silva s.n. (HAS104064); arroio Bom Jardim T2, 1-VII-2003, S.M. Alves da Silva s.n. (HAS104067); arroio Bom Jardim T1, 3-XI-2003, S.M. Alves da Silva s.n. (HAS104522); arroio Bom Jardim T1, 1-XII-2003, V.R. Werner s.n. (HAS104543); arroio Bom Jardim T2, 1-XII-2003, V.R. Werner s.n. (HAS104546); arroio Bom Jardim T1, 12-IV-2004, G.R. Cunha s.n. (HAS104783); arroio Bom Jardim T1, 9-III-2004, G.R. Cunha s.n. (HAS106095); arroio Bom Jardim T2, 7-X-2004, G.R. Cunha s.n. (HAS 106140); arroio Bom Jardim T3, 7-X-2004, G.R. Cunha s.n. (HAS106143); arroio Bom Jardim T1, 8-XI-2004, S.M. Alves da Silva s.n. (HAS106158); arroio Bom Jardim T2, 11-I-2005, G.R. Cunha s.n. (HAS106405); arroio Bom Jardim T1, 11-IV-2005, S.M. Alves da Silva s.n. (HAS106546); arroio Bom Jardim T1, 9-VIII-2005, S.M. Alves da Silva s.n. (HAS106617); arroio Bom Jardim T1, 10-X-2005, S.M. Alves da Silva s.n. (HAS106703); arroio Bom Jardim T3, 10-X-2005, S.M. Alves da Silva s.n. (HAS106709); arroio Bom Jardim T1, 8-XI-2005, S.M. Alves da Silva s.n. (HAS106716); arroio Bom Jardim T2, 8-XI-2005, S.M. Alves da Silva s.n. (HAS106718); arroio Bom Jardim T3, 8-XI-2005, S.M. Alves da Silva s.n. (HAS106721).

Distribuição no Brasil: Norte (Amazonas), Sul (Rio Grande do Sul, Santa Catarina).

Difere da variedade típica pelas menores dimensões da lórica e vista lateral triangular. Essa variedade ocorreu em temperatura da água de $11,7-26,3{ }^{\circ} \mathrm{C}$, temperatura do ar de $11,7-30{ }^{\circ} \mathrm{C}, \mathrm{pH}$ de 5,8-7,0, condutividade de 27,3-142,3 $\mu \mathrm{S} \mathrm{cm}^{-1}$, demanda bioquímica de oxigênio de 0,7-4,2 $\mathrm{mg} \mathrm{L}^{-1}$, matéria orgânica de $5,2-9,2 \mathrm{mg} \mathrm{L}^{-1}$, oxigênio dissolvido de 3,4-9,3 $\mathrm{mg} \mathrm{L}^{-1}$, sílica de 5,7-14,0 $\mathrm{mg} \mathrm{L}^{-1}$ 


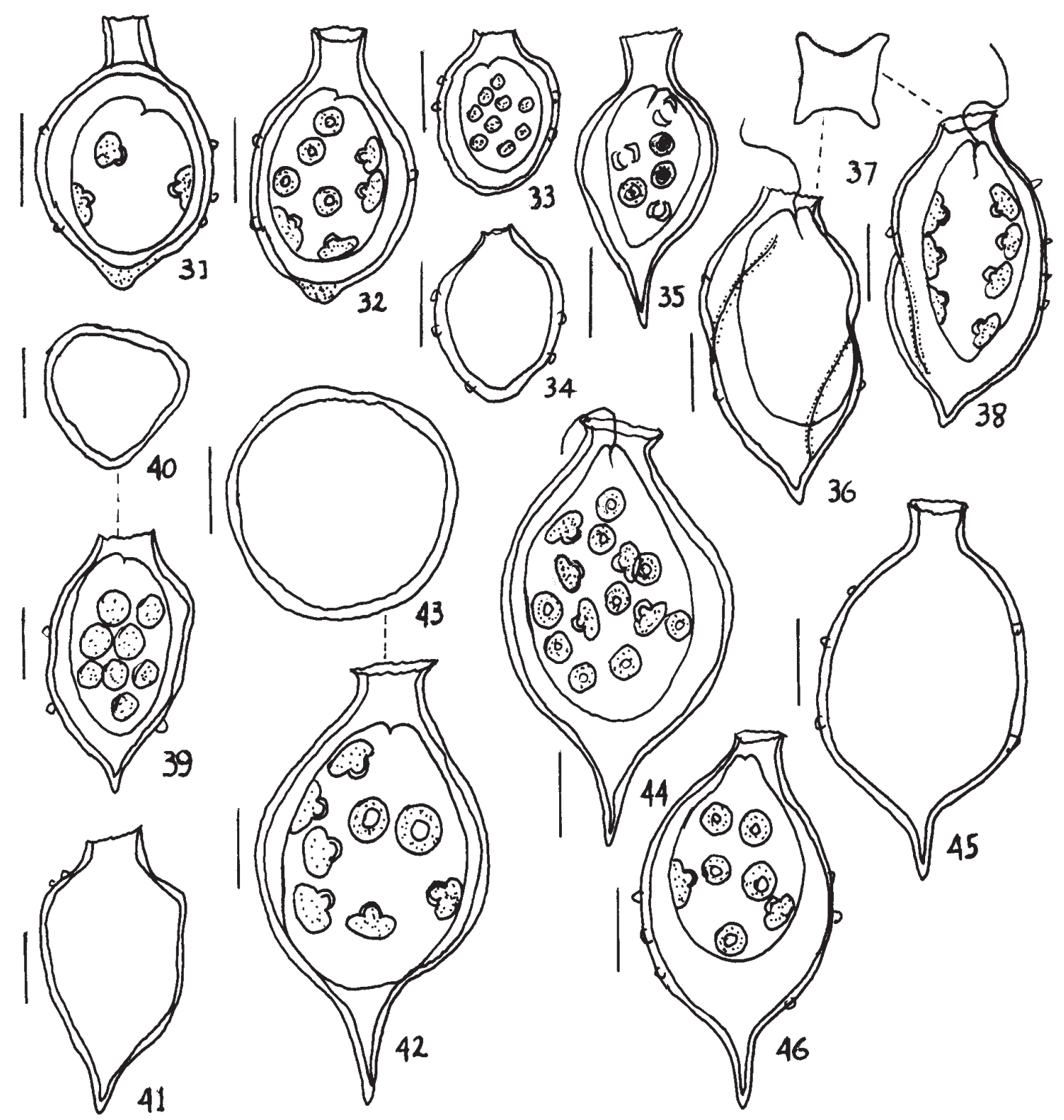

Figuras 31-46. Espécies de Strombomonas do arroio Bom Jardim, RS, Brasil. 31-32. S. scabra (Playf.) Tell \& Conf. var. ovata (Playf.) Tell \& Conf. fa. ovata (Defl.) Tell \& Conf. 33-34. S. scabra (Playf.) Tell \& Conf. var. ovata (Playf.) Tell \& Conf. fa. minor Tell \& Conf. 34. vista lateral. 35. S. schauinslandii (Lemm.) Defl. 36-38. S. tetraptera Bal. et Dast. var. gallica Bourr. \& Couté. 37. vista apical. 38-40. S. triquetra (Playf.) Defl. var. torta Rino. 40. vista apical. 43-44. S. urceolata (Stokes) Defl. 43. vista apical. 45-46. S. verrucosa (Daday) Defl. var. zmiewika (Swir.) Defl. Escala $=10 \mu \mathrm{m}$.

Figures 31-46. Species of Strombomonas from Bom Jardim Stream, RS, Brazil. 31-32. S. scabra (Playf.) Tell \& Conf. var. ovata (Playf.) Tell \& Conf. fa. ovata (Defl.) Tell \& Conf. 33-34. S. scabra (Playf.) Tell \& Conf. var. ovata (Playf.) Tell \& Conf. fa. minor Tell \& Conf. 34. lateral view. 35. S. schauinslandii (Lemm.) Defl. 36-38. S. tetraptera Bal. \& Dast. var. gallica Bourr. \& Couté. 37. apical view. 3840. S. triquetra (Playf.) Defl. var. torta Rino. 40. apical view. 43-44. S. urceolata (Stokes) Defl. 43. apical view. 45-46. S. verrucosa (Daday) Defl. var. zmiewika (Swir.) Defl. Scale $=10 \mu \mathrm{m}$. 
e ortofosfato de 10,0-1120,0 $\mu \mathrm{g} \mathrm{L}^{-1}$. Pode ser considerada uma espécie euritérmica por suportar amplitude de temperatura superior a $10^{\circ} \mathrm{C}$.

Strombomonas schauinslandii (Lemm.) Defl., Arch. Protistenk. 69(3): 594, fig. 90-96. 1930.

Figura 35

Lórica napiforme, 33-35 $\mu \mathrm{m}$ compr., 14,5-15 $\mu \mathrm{m}$ larg., $\mathrm{Rc} / 1=2,3-2,4$.

Material examinado: BRASIL. Rio GRANDE Do Sul: Triunfo, arroio Bom Jardim T3, 6-II-2002, M.L. Nunes s.n. (HAS103195); arroio Bom Jardim T1, 6-II-2002, M.L. Nunes s.n. (HAS103202); arroio Bom Jardim T3, 22-IV-2002, S.M. Alves da Silva s.n. (HAS103397).

Distribuição no Brasil: Norte (Amazonas), CentroOeste (Mato Grosso do Sul), Sul (Paraná, Rio Grande do Sul).

Foi observada a presença de duplopirenoides, estrutura não registrada anteriormente o que amplia a circunscrição da espécie. Na Argentina foram encontradas também em clima subtropical (Tell $\&$ Conforti 1986). Essa variedade ocorreu em temperatura da água de $11,7-26,3{ }^{\circ} \mathrm{C}$, temperatura do ar de $21,5-30^{\circ} \mathrm{C}$, $\mathrm{pH}$ de $6,8-6,9$, condutividade de $113,6-260,6 \mu \mathrm{S} \mathrm{cm}^{-1}$, demanda bioquímica de oxigênio de $2,2 \mathrm{mg} \mathrm{L}^{-1}$, matéria orgânica de 5,8-8,7 $\mathrm{mg} \mathrm{L}^{-1}$, oxigênio dissolvido de $5,8-8,7 \mathrm{mg} \mathrm{L}^{-1}$, sílica de $9,8 \mathrm{mg} \mathrm{L}^{-1}$ e ortofosfato de $230,0 \mu \mathrm{g} \mathrm{L}^{-1}$.

Strombomonas tetraptera Bal. et Dast. var. gallica Bour. \& Couté, Rev. Algol. 13(4): 299, fig. 17. 1978.

Figuras 36-38

Lórica de contorno geral elíptica turbinada, 37-40 $\mu \mathrm{m}$ compr., 19-24 $\mu \mathrm{m}$ larg., $\mathrm{Rc} / 1=1,7-1,9$; vista polar quadrangular, lados côncavos e ângulos arredondados.

Material examinado: BRASIL. Rio GRANDE do Sul: Triunfo, arroio Bom Jardim T1, 6-II-2002, M.L. Nunes s.n. (HAS103202); arroio Bom Jardim T3, 1-XII-2003, V.R. Werner s.n. (HAS104549); arroio Bom Jardim T3, 14-III-2005, S.M. Alves da Silva s.n. (HAS106449); arroio Bom Jardim T2, 9-I-2006, S.M. Alves da Silva s.n. (HAS106770).

Distribuição no Brasil: Centro-Oeste (Mato Grosso, como S. tetraptera), Sul (Rio Grande do Sul).

Segundo Bourrelly \& Coute (1978), essa variedade difere da espécie típica pela torção regular helicóide da lórica, pelas menores dimensões e pela vista apical quadrangular. Os exemplares apresentaram-se ligeiramente maiores em largura que os dados pelos autores. Considera-se que o material encontrado por Menezes e Fernandes (1987) no município de Cáceres, no Mato Grosso, trata-se da var. gallica e não da espécie típica. Essa variedade ocorreu em temperatura da água de $22,3-24,6^{\circ} \mathrm{C}$, temperatura do ar de $22,1-30,0^{\circ} \mathrm{C}, \mathrm{pH}$ de $6,8-7,1$, condutividade de $82,5-278 \mu \mathrm{S} \mathrm{cm}^{-1}$, demanda bioquímica de oxigênio de 1,0-1,4 mg L-1, matéria orgânica de 5,8-9,0 $\mathrm{mg} \mathrm{L}^{-1}$, oxigênio dissolvido de $3,4-9,3 \mathrm{mg} \mathrm{L}^{-1}$, sílica de 10,9-15,3 $\mathrm{mg} \mathrm{L}^{-1}$ e ortofosfato de 50,0-460,0 $\mu \mathrm{g} \mathrm{L}^{-1}$.

Strombomonas triquetra (Playf.) Defl. var. torta Rino, Rev. Ciênc. Biol. 5: 169, pl. 9, fig. 7-10. 1972. Figuras 38-40

Lórica levemente triangular a trapezoidal, 36-41 $\mu \mathrm{m}$ compr., 17-21 $\mu \mathrm{m}$ larg., Rc/1 = 1,8-1,9.

Material examinado: BRASIL. Rio GRANDE DO Sul: Triunfo, arroio Bom Jardim T1, 3-VII-2002, S.M. Alves da Silva s.n. (HAS103457); arroio Bom Jardim T1, 14-XI-2002, S.M. Alves da Silva s.n. (HAS103665); arroio Bom Jardim T3, 14-XI-2002, S.M. Alves da Silva s.n. (HAS103671); arroio Bom Jardim T2, 10-III-2003, S.M. Alves da Silva s.n. (HAS103949); arroio Bom Jardim T2, 3-XI-2003, S.M. Alves da Silva s.n. (HAS 104525); arroio Bom Jardim T1, 5-I-2004, S.M. Alves da Silva s.n. (HAS104561); arroio Bom Jardim T3, 5-I-2004, S.M. Alves da Silva s.n. (HAS104567); arroio Bom Jardim T1, 11-V-2004, S.M. Alves da Silva s.n. (HAS104804); arroio Bom Jardim T2, 8-XI-2004, S.M. Alves da Silva s.n. (HAS106161); arroio Bom Jardim T3, 15-II-2005, S.M. Alves da Silva s.n. (HAS106429); arroio Bom Jardim T3, 14-III-2005, S.M. Alves da Silva s.n. (HAS106449); arroio Bom Jardim T2, 14-III-2005, S.M. Alves da Silva s.n. (HAS106446); arroio Bom Jardim T3, 8-XI-2005, S.M. Alves da Silva s.n. (HAS106721).

Distribuição no Brasil: Nordeste (Paraíba), Sul (Rio Grande do Sul).

Possui vista apical triangular com ângulos arredondados e apresentou haplopirenóides. Segundo Tell \& Conforti (1986) essa variedade foi registrada em lugares quentes de Moçambique e Argentina. No Rio Grande do Sul apesar de ser encontrada em clima subtropical ocorreu em temperatura da água oscilando entre $17-30,3{ }^{\circ} \mathrm{C}$ (Alves-da-Silva \& Bridi 
1994) e presentemente em temperaturas da água entre 17-29, $1{ }^{\circ} \mathrm{C}$, podendo ser considerada uma espécie euritérmica. Essa variedade ocorreu em temperatura do ar de $11,5-29,1^{\circ} \mathrm{C}, \mathrm{pH}$ de 5,6-7,0, condutividade de $13,2-278,0 \mu \mathrm{S} \mathrm{cm}^{-1}$, demanda bioquímica de oxigênio de 0,5-4,4 $\mathrm{mg} \mathrm{L}^{-1}$, matéria orgânica de 3,9-9,0 $\mathrm{mg} \mathrm{L}^{-1}$, oxigênio dissolvido de $3,7-8,2 \mathrm{mg} \mathrm{L}^{-1}$, sílica de 7,4-13,2 $\mathrm{mg} \mathrm{L}^{-1}$ e ortofosfato de 30,0-520,0 $\mu \mathrm{g} \mathrm{L}^{-1}$.

Strombomonas urceolata (Stokes) Defl., Arch. Protistenk. 69(3): 586-587, fig. 70,71-73. 1930.

Figuras 41-43

Lórica urceolada, 50-53 $\mu \mathrm{m}$ comp., 27-28 $\mu \mathrm{m}$ larg., $\mathrm{Rc} / 1=1,8-2,0$.

Material examinado: BRASIL. Rio GRANDE Do Sul: Triunfo, arroio Bom Jardim T2, 6-II-2002, M.L. Nunes s.n. (HAS103188); arroio Bom Jardim T1, 6-II-2002, M.L. Nunes s.n. (HAS103202); arroio Bom Jardim T2, 8-XI-2004, S.M. Alves da Silva s.n. (HAS106161); arroio Bom Jardim T2, 6-XII-2004, S.M. Alves da Silva s.n. (HAS106390); arroio Bom Jardim T3, 14-III-2005, S.M. Alves da Silva s.n. (HAS106449); arroio Bom Jardim T1, 14-XI-2002, S.M. Alves da Silva s.n. (HAS103665); arroio Bom Jardim T1, 8-XI-2005, S.M. Alves da Silva s.n. (HAS 106716); arroio Bom Jardim T2, 9-I-2006, S.M. Alves da Silva s.n. (HAS106770); arroio Bom Jardim T3, 8-II-2006, S.M. Alves da Silva s.n. (HAS106786).

Distribuição no Brasil: Norte (Amazonas), CentroOeste (Mato Grosso), Sudeste (Rio de Janeiro), Sul (Paraná, Rio Grande do Sul).

Possui pólo anterior atenuado abruptamente em colarinho curto, subcilíndrico, alargado na região distal, com 3-5 $\mu \mathrm{m}$ alt. e 7,5-8 $\mu \mathrm{m}$ larg., com haplopirenóides presentes. Foi observado flagelo ca. $1 / 5$ do comprimento da lórica. Essa variedade ocorreu em temperatura da água de $20,6-26,3{ }^{\circ} \mathrm{C}$, temperatura do ar de $20,8-30,0{ }^{\circ} \mathrm{C}$, $\mathrm{pH}$ de $5,8-6,9$, condutividade de 13,2-278 $\mu \mathrm{S} \mathrm{cm}^{-1}$, demanda bioquímica de oxigênio de 1,0-2,6 $\mathrm{mg} \mathrm{L}^{-1}$, matéria orgânica de 5,8-10,2 $\mathrm{mg} \mathrm{L}^{-1}$, oxigênio dissolvido de 4,5-7,2 $\mathrm{mg} \mathrm{L}^{-1}$, sílica de 8,2-15,3 $\mathrm{mg} \mathrm{L}^{-1}$ e ortofosfato de $30,0-460,0 \mu \mathrm{g} \mathrm{L} \mathrm{L}^{-1}$.

Strombomonas verrucosa (Daday) Defl. var. zmiewika (Swir.) Defl., Arch. Protistenk. 69(3): 586587, fig. 70,71-73. 1930.

Figuras $44-45$

Lórica ovada a trapezoidal, 37-53 $\mu \mathrm{m}$ compr., 20-31 $\mu \mathrm{m}$ larg., $\mathrm{Rc} / \mathrm{l}=1,7-2,0$.
Material examinado: BRASIL. Rio GRANDE do Sul: Triunfo, arroio Bom Jardim T1, 14-I-2002, S.M. Alves da Silva s.n. (HAS103163); arroio Bom Jardim T2, 6-II-2002, M.L. Nunes s.n. (HAS103188); arroio Bom Jardim T3, 6-II-2002, M.L. Nunes s.n. (HAS103195); arroio Bom Jardim T1, 6-II-2002, M.L. Nunes s.n. (HAS103202); arroio Bom Jardim T2, 25-III-2002, S.M. Alves da Silva s.n. (HAS103250); arroio Bom Jardim T3, 25-III-2002, S.M. Alves da Silva s.n. (HAS103254); arroio Bom Jardim T1, 22-IV-2002, S.M. Alves da Silva s.n. (HAS103391); arroio Bom Jardim T3, 22-IV-2002, S.M. Alves da Silva s.n. (HAS103397); arroio Bom Jardim T1, 3-VII-2002, S.M. Alves da Silva s.n. (HAS 103457); arroio Bom Jardim T1, 14-XI-2002, S.M. Alves da Silva s.n. (HAS103665); arroio Bom Jardim T2, 14-XI-2002, S.M. Alves da Silva s.n. (HAS103668); arroio Bom Jardim T3, 14-XI-2002, S.M. Alves da Silva s.n. (HAS103671); arroio Bom Jardim T2, 2-XII-2002, S.M. Alves da Silva s.n. (HAS 103693); arroio Bom Jardim T3, 2-XII-2002, S.M. Alves da Silva s.n. (HAS103696); arroio Bom Jardim T2, 6-I-2003, G.R. Cunha s.n. (HAS103833); arroio Bom Jardim T3, 10-III-2003, S.M. Alves da Silva s.n. (HAS103952); arroio Bom Jardim T1, 4-II-2003, G.R. Cunha s.n. (HAS103856); arroio Bom Jardim T2, 4-II-2003, G.R. Cunha s.n. (HAS103859); arroio Bom Jardim T3, 4-II-2003, G.R. Cunha s.n. (HAS103862); arroio Bom Jardim T2, 10-III-2003, S.M. Alves da Silva s.n. (HAS103949); arroio Bom Jardim T1, 5-V-2003, G.R. Cunha s.n. (HAS104014); arroio Bom Jardim T2, 5-V-2003, G.R. Cunha s.n. (HAS104017); arroio Bom Jardim T3, 2-VI-2003, S.M. Alves da Silva s.n. (HAS104045); arroio Bom Jardim T2, 1-VII-2003, S.M. Alves da Silva s.n. (HAS104067); arroio Bom Jardim T3, 4-VIII-2003, S.M. Alves da Silva s.n. (HAS104283); arroio Bom Jardim T3, 1-XII-2003, V.R. Werner s.n. (HAS 104549); arroio Bom Jardim T3, 5-I-2004, S.M. Alves da Silva s.n. (HAS104567); arroio Bom Jardim T3, 3-II-2004, S.M. Alves da Silva s.n. (HAS104588); arroio Bom Jardim T2, 7-VI-2004, S.M. Alves da Silva s.n. (HAS104824); arroio Bom Jardim T1, 9-III-2004, G.R. Cunha s.n. (HAS106095); arroio Bom Jardim T3, 7-X-2004, G.R. Cunha s.n. (HAS106143); arroio Bom Jardim T3, 8-XI-2004, S.M. Alves da Silva s.n. (HAS106164); arroio Bom Jardim T2, 8-XI-2004, S.M. Alves da Silva s.n. (HAS106161); arroio Bom Jardim T2, 6-XII-2004, S.M. Alves da Silva s.n. (HAS106390); arroio Bom Jardim T3, 6-XII-2004, S.M. Alves da 
Silva s.n. (HAS106393); arroio Bom Jardim T3, 15-II-2005, S.M. Alves da Silva s.n. (HAS106429); arroio Bom Jardim T2, 14-III-2005, S.M. Alves da Silva s.n. (HAS106446); arroio Bom Jardim T3, 14-III-2005, S.M. Alves da Silva s.n. (HAS106449); arroio Bom Jardim T3, 11-IV-2005, S.M. Alves da Silva s.n. (HAS106552); arroio Bom Jardim T3, 12-V-2005, G.R. Cunha s.n. (HAS106565); arroio Bom Jardim T1, 10-X-2005, S.M. Alves da Silva s.n. (HAS106703); arroio Bom Jardim T2, 8-XI-2005, S.M. Alves da Silva s.n. (HAS106718); arroio Bom Jardim T3, 8-XI-2005, S.M. Alves da Silva s.n. (HAS106721); arroio Bom Jardim T3, 5-XII-2005, V.R. Werner s.n. (HAS106737); arroio Bom Jardim T2, 9-I-2006, S.M. Alves da Silva s.n. (HAS106770); arroio Bom Jardim T2, 8-II-2005, S.M. Alves da Silva s.n. (HAS106783); arroio Bom Jardim T3, 8-II-2006, S.M. Alves da Silva s.n. (HAS106786); arroio Bom Jardim T2, 13-IX-2006, S.E. Salomoni s.n. (HAS107134); arroio Bom Jardim T3, 12-XII-2006, S.E. Salomoni s.n. (HAS107257).

Distribuição no Brasil: Norte (Amazonas, Pará), Centro-Oeste (Mato Grosso do Sul), Sudeste (Rio de Janeiro), Sul (Rio Grande do Sul).

Essa variedade ocorreu em temperatura da água de $14,5-33,6^{\circ} \mathrm{C}$, temperatura do ar de $16,5-30,0{ }^{\circ} \mathrm{C}$, $\mathrm{pH}$ de 5,6-7,1, condutividade de 13,2-1.052,0 $\mu \mathrm{S} \mathrm{cm}^{-1}$, demanda bioquímica de oxigênio de $0,3-5,4 \mathrm{mg} \mathrm{L}^{-1}$, matéria orgânica de 5,3-11,3 $\mathrm{mg} \mathrm{L}^{-1}$, oxigênio dissolvido de 4,3-9,0 $\mathrm{mg} \mathrm{L}^{-1}$, sílica de 5,1-15,3 $\mathrm{mg} \mathrm{L}^{-1} \mathrm{e}$ ortofosfato de 10,0-950,0 $\mu \mathrm{g} \mathrm{L}^{-1}$. Pode ser considerada uma espécie euritérmica por suportar amplitude de temperatura superior a $10{ }^{\circ} \mathrm{C}$.

O estudo de Strombomonas faz parte de um projeto mais amplo cujo objetivo é realizar o estudo da estrutura da comunidade fitoplanctônica do arroio Bom Jardim resultado da parceria entre a Braskem e Fundação Zoobotânica do Rio Grande do Sul. Nesse ambiente, no período de 2002 a 2006, foi observado que o número de táxons de Euglenophyta em amostras quantitativas foi próximo a metade do total de espécies identificadas (51\%) em amostras qualitativas. $\mathrm{Na}$ análise quantitativa foram identificados somente cinco táxons de Strombomonas, enquanto na análise qualitativa foram registradas 26 espécies e infraespécies desse gênero. Igual número de Strombomonas registrado por Pérez (2002) em estudo qualitativo e quantitativo do fitoplâncton no rio Negro, no Uruguai, um ecossistema mesotrófico com tendência a eutrofização nos meses de verão. Neste ambiente foram registrados 60 táxons de
Euglenophyceae. Embora a autora não tenha especificado quais espécies foram encontradas nas duas análises, supõe-se que grande maioria foi registrada em amostras qualitativas, visto que este grupo de algas no país tem sua maior ocorrência em amostras coletadas com rede de plâncton (e.g. Cecy 1990, Conforti 1993, Jati \& Train 1995, Alves-daSilva \& Bridi 2004, Alves-da-Silva \& Bicudo 2006, 2009).

A riqueza de táxons de Strombomonas nos locais amostrados pode ser considerada relativamente alta (26 táxons) quando comparada com outros trabalhos no país. No Brasil as únicas publicações que mencionam riqueza superior à do presente estudo são Conforti (1993) - (43 táxons) e Alves-da-Silva \& Bridi (2004) - (34 táxons). Apesar do alto número de espécies, foram identificadas Strombomonas em somente $54,9 \%$ do total das amostras analisadas (175), e na maioria das amostras ocorreram poucos indivíduos por lâminas (menos de cinco), resultado semelhante aos citados por Alves-da-Silva \& Bridi (2004) e Alves-da-Silva \& von Kurrle (2009).

Quanto à avaliação biogeográfica, oito táxons que ocorreram no arroio possuem distribuição cosmopolita (Strombomonas fluviatilis, S. fluviatilis var. levis, $S$. gibberosa var. gibberosa, $S$. girardiana var. girardiana, S. maxima, S. scabra var. scabra, S. schauinslandii e $S$. urceolata). Os 18 táxons restantes apresentam distribuição restrita a dois ou três continentes.

No Brasil, Strombomonas ensifera, S. fluviatilis var. fluviatilis, $S$. scabra var. longicollis, $S$. urceolata $\mathrm{e}$ S. verrucosa var. zmiewika destacam-se por apresentar ampla distribuição geográfica. Com relação ao Estado do Rio Grande do Sul, 23 táxons já haviam sido registrados anteriormente. Strombomonas eurystoma f. incurva e $S$. recurvata são novos registros para o país e $S$. brevicaudata é citada pela primeira vez para o Estado e pela segunda vez no Brasil.

Quanto à frequência de ocorrência, a única variedade constante foi $S$. verrucosa var. zmiewika por ter ocorrido em 52\% das 96 amostras em que ocorreu o gênero. Seis táxons foram comuns (entre $10 \%$ e 50\%), sendo eles: Strombomonas borystheniensis, S. girardiana var. girardiana, S. scabra var. scabra, S. scabra var. intermedia, S. scabra var. ovata f. minor e $S$. triquetra var. torta. Os demais 19 táxons foram considerados raros por estarem presentes em $<10 \%$ das amostras.

A análise de agrupamento qualitativo, tendo como base a presença e ausência de espécies identificadas, 
e utilizando como medida de similaridade o índice de Jaccard, indicou que a maior similaridade ocorreu entre os trechos T2 (Montante ao Sitel) e T3 (Frente ao Sitel), igual a $82 \%$, diferentemente da nascente (T1) que apresentou um valor de 73\% (Figura 47). Esta diferença poderia ser explicada considerando que no trecho T1 houve a presença exclusiva das espécies $S$. brevicaudata, S. scabra var. labiata, S. rotunda. É importante destacar que a espécie exclusiva do T1, S. brevicaudata, corresponde ao primeiro registro para o Estado do Rio Grande do Sul e segundo para o Brasil. É possível inferir que há uma alta probabilidade de que a ocorrência exclusiva dessas espécies neste trecho do rio esteja vinculada a concentração de matéria orgânica da água $\left(7,4\right.$ - 7,8 $\left.\mathrm{mg} \mathrm{L}^{-1}\right)$, caracterizando condições mesossapróbicas de poluição segundo classificação de Hamm (1969) e confirmando a hipótese de que muitas espécies dessa divisão apresentam preferências ecológicas para águas dessa natureza. Por exemplo, Sladecék (1973) cita três táxons de Strombomonas indicadoras de condições $\beta$ - mesossapróbicas, enquanto Alves-da-Silva et al. (2007) registraram oito espécies desse gênero nas mesmas condições de saprobidade, no Parque Estadual Delta do Jacuí, RS.

Essa diferença poderia ser explicada, ainda, considerando que no trecho $\mathrm{T} 1$ verificou-se a menor correnteza e profundidade $(<30 \mathrm{~cm})$ quando comparado com as verificadas nos trechos T2 e T3.

Essas espécies apresentam também distribuição mundial restrita, considerando que $S$. brevicaudata foi registrada somente na América do Norte e do Sul; enquanto que $S$. scabra var. labiata na América do Sul, Ásia e Oceania; e $S$. rotunda na América do Norte, América do Sul, Ásia e Oceania. O T2 não

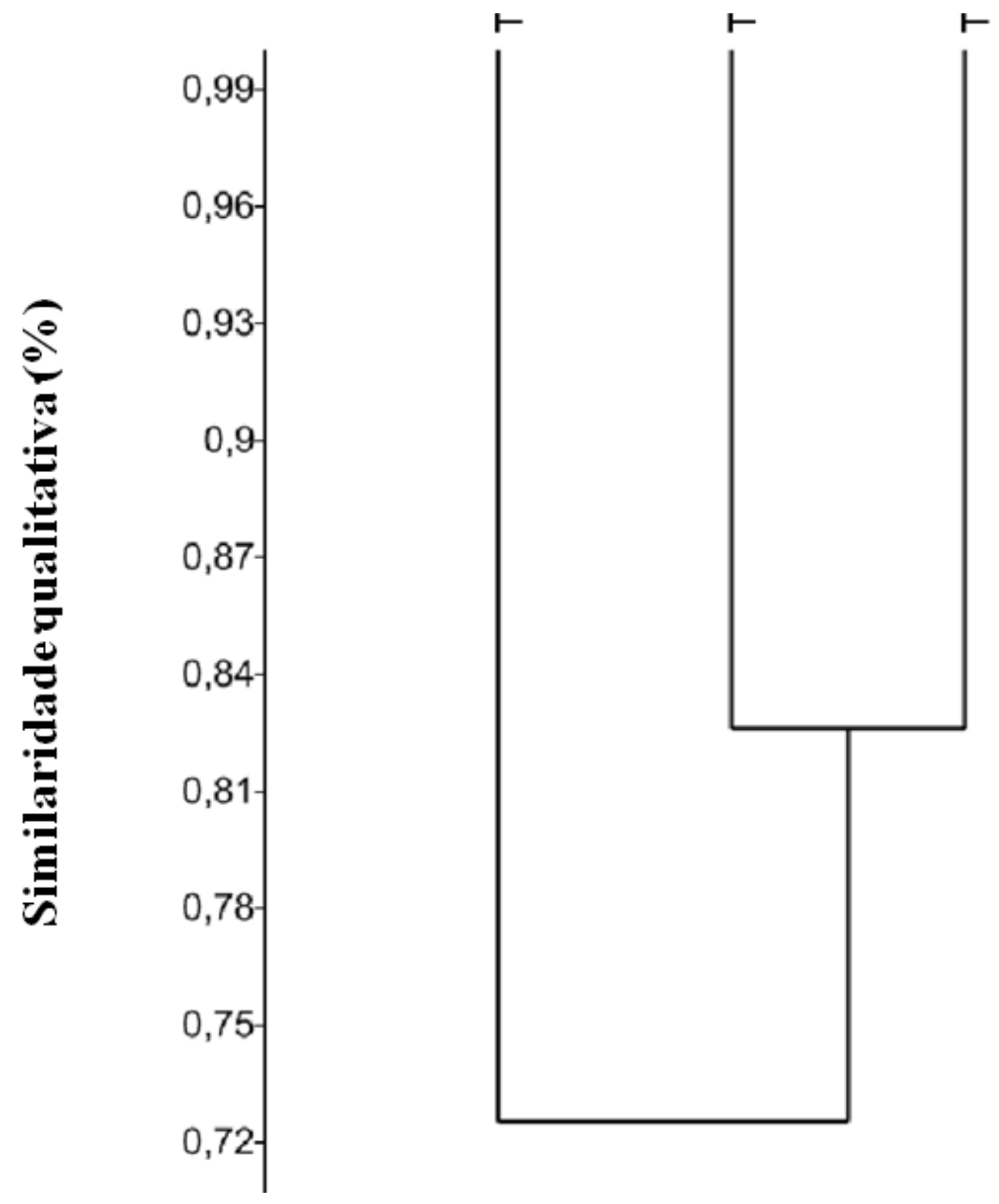

Figura 47. Analise de agrupamento qualitativo (Jaccard, \%), de espécies e infraespécies de Strombomonas em três trechos do arroio Bom Jardim, RS, Brasil. Onde, T1: Nascente, T2: Montante ao Sitel e T3: Frente ao Sitel.

Figure 47. Qualitative cluster analysis (Jaccard,\%) of the species and infraespecies of Strombomonas in three reaches from Bom Jardim Stream, RS, Brazil. Where, T1: Spring, T2: Upstream of Sitel, and T3: Front of Sitel. 
apresentou nenhuma espécie exclusiva, enquanto que o T3 apresentou uma espécie exclusiva, S. girardiana var. triondulata. Quinze espécies e infraespécies foram comuns aos três trechos do arroio Bom Jardim.

A riqueza oscilou de um a 17 táxons por amostra, com predomínio de número de táxons por amostra muito baixos (menores que cinco), sendo observada variação espacial e temporal, sem configurar um padrão sazonal. Em relação à variação espacial, a nascente foi o trecho que apresentou a maior riqueza no período de 2002-2006. Quanto à variação temporal, as maiores riquezas foram verificadas no verão e primavera (fevereiro, novembro e setembro de 2002), onde foram registrados 17,13 , e nove táxons, respectivamente. Nestes três meses as condições ambientais da água foram temperatura da água $\left(16,2-24,6^{\circ} \mathrm{C}\right)$, temperatura do ar $\left(13,6-30,5^{\circ} \mathrm{C}\right)$, $\mathrm{pH}(6,8)$, condutividade $\left(13,2-113,6 \mu \mathrm{S} \mathrm{cm} \mathrm{cm}^{-1}\right)$, demanda bioquímica de oxigênio $\left(2,0-2,1 \mathrm{mg} \mathrm{L}^{-1}\right)$, matéria orgânica $\left(5,8-7,8 \mathrm{mg} \mathrm{L}^{-1}\right)$, oxigênio dissolvido $\left(5,8-8,5 \mathrm{mg} \mathrm{L}^{-1}\right)$, sílica $\left(7,5-12,3 \mathrm{mg} \mathrm{L}^{-1}\right) \mathrm{e}$ ortofosfato ( 40,0-640,0 $\mu \mathrm{g} \mathrm{L}^{-1}$ ).

Quanto à sílica, as maiores riquezas foram registradas em concentrações maiores que $10,0 \mathrm{mg} \mathrm{L}^{-1}$, tal como verificado por Alves-da-Silva \& Bridi (2004) no Delta do Jacuí, no Rio Grande do Sul. Estas autoras registraram maiores riquezas do gênero em condições semelhantes às registradas no arroio Bom Jardim quanto à temperatura, $\mathrm{pH}$, matéria orgânica e demanda bioquímica de oxigênio. Segundo Conforti et al. (1994), as lóricas das Strombomonas são formadas primeiramente por sílica e ferro, sendo estes dois elementos importantes na sua constituição. No arroio, em $94 \%$ das amostras a concentração da sílica foi maior que $10,0 \mathrm{mg} \mathrm{L}^{-1}$, concentrações altas segundo Garrel \& Mackenzie (1971), o que poderia explicar em parte a ocorrência de tantas espécies do gênero registradas neste ambiente.

O ano com a menor distribuição de Strombomonas foi 2006, ocorrendo em apenas quatro meses do ano, e somente nos trechos T2 e T3. Essa menor ocorrência poderia ser explicada considerando a baixa precipitação pluviométrica registrada, altas temperaturas excepcionais nos meses de inverno e primavera, altas concentrações de condutividade e baixa concentração de oxigênio dissolvido, ortofosfato e sílica. Além destas variáveis, a falta de correnteza e baixa profundidade da água na nascente $(10,0-30,0 \mathrm{~cm}-23,2 \pm 8,2 \mathrm{~cm})$, podem ter influenciado na inexistência de representantes do gênero nesse trecho.
Cabe ressaltar que apesar do alto número de táxons de Strombomonas registradas no arroio Bom Jardim, $73 \%$ apresentaram baixa freqüência de ocorrência, sendo necessários maiores estudos para elucidar quais são as condições abióticas requeridas pelo gênero. Somente a ampliação de um maior número de estudos qualitativos e ecológicos em diferentes ambientes aquáticos propiciará à compreensão da sua distribuição e conhecimento desse gênero tão pouco conhecido em nível mundial.

\section{Agradecimentos}

À Fundação de Amparo à Pesquisa do Estado do Rio Grande do Sul pelas Bolsas de Iniciação Científica de Josemari da Cruz Cabreira (Proc. $\mathrm{n}^{\mathrm{o}}$ 11/0418-6) e Karina Federle Consoni (Proc. $\mathrm{n}^{\mathrm{o}}$ 08511100); à BRASKEM, pelo apoio logístico durante as coleta de amostras; aos colegas da Seção de Botânica de Criptógamas do Museu de Ciências Naturais (MCN) da Fundação Zoobotânica do Rio Grande do Sul (FZBRS), pelo auxílio nas expedições de coletas; a Manoel Luis Nunes, pelas análises químicas; e a Ricardo Aranha Ramos do Laboratório de Geoprocessamento da FZBRS; pela confecção do mapa.

\section{Literatura citada}

Alves-da-Silva, S.M. 1988. Euglenaceae pigmentadas (Euglenophyta) da Estação Ecológica do Taim, Rio Grande do Sul, Brasil. Iheringia, série Botânica 38: $109-126$

Alves-da-Silva, S.M. \& Bicudo, C.E.M. 2003. Strombomonas carinata sp.nov. and Strombomonas ferrazi sp.nov., two new Euglenophyceae from the state of Rio Grande do Sul, southern Brazil. Acta Botanica Brasilica 17: 147-154.

Alves-da-Silva, S.M. \& Bicudo, C.E.M. 2006. Contribution to the knowledge of genus Strombomonas (Euglenophyceae) of the state of Rio Grande do Sul, southern Brazil. Algological Studies 119: 29-58.

Alves-da-Silva, S.M. \& Bicudo, C.E.M. 2009. Cryptoglena, Monomorphina and Phacus (Euglenophyceae) of a reservoir in the State of Rio Grande do Sul, southern Brazil. Revista Brasileira de Botanica 32: 253-270.

Alves-da-Silva, S.M., Bicudo, C.E.M., Consoni, K.F., Silva, L.S. \& Borges, C.L.L. 2011. Genus Lepocinclis (Euglenophyceae) along five years in the area of influence of the Southern Petrochemical Pole, Rio Grande do Sul State, Brazil. Hoehnea 38: 257-272. 
Alves-da-Silva, S.M. \& Bridi, F.C. 2004. Euglenophyta no Parque Estadual do Jacuí, Rio Grande do Sul, Sul do Brasil. 3. Gênero Strombomonas Defl. Acta Botanica Brasilica 18: 555-572.

Alves-da-Silva, S.M. \& Fortuna, J.R. 2006. Euglenophyceae de ambientes lênticos na planície costeira do Rio Grande do Sul, sul do Brasil: gêneros Euglena Ehr. e Lepocinclis Perty. Acta Botanica Brasilica 20: 411-422.

Alves-da-Silva, S.M. \& Hahn, A.T. 2004. Study of Euglenophyta in the Jacuí Delta State Park, Rio Grande do Sul, Brazil. 1. Euglena Ehr., Lepocinclis Perty. Acta Botanica Brasilica 18: 123-140.

Alves-da-Silva, S.M., Hermany, G. \& Oliveira, M.A. 2007. Diversity and ecological considerations on pigmented Euglenophyceae in the State Park of The Jacuí Delta, Rio Grande do Sul, Southern Brazil. Biociências 15: 8-20.

Alves-da-Silva \& von Kurrle, I.L.P. 2009. O gênero Strombomonas (Euglenophyceae pigmentadas) em ambientes lênticos na Planície Costeira do Rio Grande do Sul, Brasil. Acta Botanica Brasilica 23: 944-955.

Alves-da-Silva, S.M. \& Torres, J. R. 1994a. O gênero Strombomonas no Parque Zoológico, Sapucaia do Sul e no Jardim Botânico, Porto Alegre, Rio Grande do Sul, Brasil. Iheringia, série Botânica 44: 15-32.

Alves-da-Silva, S.M. \& Torres, J.R. 1994b. Estudo Taxonômico do gênero Phacus Duj. (Euglenaceae) no Parque Zoológico, Sapucaia do Sul e no Jardim Botânico, Porto Alegre, Rio Grande do Sul. Iheringia, série Botânica 44: 45-83.

American Public Health Association. 1992. Standards methods for the examination of water and waste water. 18 ed. Washington.

Araújo, G.J.M., Barbosa, J.E.L. \& Barbosa, L.G. 2012. Pigmented Euglenophytes in a natural and shallow lake in the semiarid region of Paraíba State, Brazil. Brazilian Journal of Botany 35: 17-30.

Bennett, M.S. \& Triemer, R.E. 2012. A new method for obtaining nuclear gene sequences from field samples and taxonomic revisions of the photosynthetic euglenoids Lepocinclis (Euglena) helicoideus and Lepocinclis (Phacus) horridus (Euglenophyta). Journal of Phycology 48: 254-260.

Bittencourt-Oliveira, M. 1997. Euglenophyceae do Reservatório de Balbina, Estado do Amazonas, Brasil. Hoehnea 24: 21-35.

Bourrelly, P.C. 1970. Les algues d'eau douce: initation à la systematique, III. Les algues blues et rouges, les Eugléniens, Peridiniens et Cryptomonadines. N. Boubée, Paris.

Bourrelly, P.C.\& Couté, A. 1978. Alges d'eau douce rares ou nouvelles pour la flore française. Revue Algologique 13: 295-307.
Brosnan, S., Shin, W., Kjer, K.M. \& Triemer, R.E. 2003. Phylogeny of the photosynthetic euglenophytes inferred from the nuclear SSU and partial LSU rDNA. International Journal of Systematic and Evolutionary Microbiology 53: 1175-1186.

Brosnan, S., Brow P.J.P., Farmer, M.A. \& Triemer, R.E. 2005. Morphological separation of the Euglenoid genera Trachelomonas and Strombomonas (Euglenophyta) based on lorica development and posterior strip reduction. Journal of Phycology 41: 590-605.

Cecy, I.T. 1990. A Restinga do Pontal do Sul, município de Paranaguá, Pr. I- Levantamento ficológico (Euglenophyta) e físico-químico. Arquivos de Biologia e Tecnolologia 33: 1-79.

Ciugulea, I., Nudelman, M.A., Brosnan, S. \& Triemer, R.E. 2008. Phylogeny of the Euglenoid loricate genera Trachelomonas and Strombomonas (Euglenophyta) inferred from nuclear SSU and LSU rDNA. Journal of Phycology 44: 406-418.

Conforti, V.T.D. 1993. Study of the Euglenophyta from Camaleão lake (Amazonas-Brazil), II. Strombomonas Defl. Revue Hydrobiological Tropical 26: 187-197.

Conforti, V.T.D. \& Joo, G. 1994. Taxonomic and ultrastructural study of Trachelomonas Ehr. and Strombomonas Defl. (Euglenophyta) from Oxbow lakes in Alabama and Indiana (U.S.A.). Cryptogamie, Algologique 15: 267-286.

Confort, V.T.D. 2009. Floristic and ultrastructural study of the genus Strombomonas (Euglenophyta) from New Jersey fresh Waters. Algological Studies 132: 1-20.

Conforti, V.T.D. \& Zalocar Y.D. 2003. Ultrastructural study of Strombomonas (Euglenophyta) from the province of Chaco (Argentina). Boletin de la Sociedad Argentina de Botanica 38: 5-12.

Conforti, V.T.D., Walne, P.L. \& Dunlap, J.R. 1994. Comparative ultrastructure and elemental composition of envelopes of Trachelomonas and Strombomonas (Euglenophyta). Acta Protozoologica 33: 71-78.

Conti, J.B. \& Furlan, S.A. 2003. Geoecologia: o clima, os solos e a biota. pp. 67-207. In: J.L. Ross (org.). Geografia do Brasil, editora da Universidade de São Paulo, São Paulo.

Conrad, W. \& Van-Meel, L. 1952. Matériaux por une monographie de Trachelomonas Ehrenberg, C. 1834. Strombomonas Deflandre, G. 1930, et Euglena Ehrenberg. C. 1832, genres d'Euglénacées. Mémoires d l'Institute Royal des sciences naturelle Belgique 124: 1-176.

Deflandre, G. 1930. Strombomonas, nouveaux genre d'Euglenacées (Trachelomonas Ehr. pro parte) Archiv für Protistenkunde 69: 551-614.

Dillard, G.E. 2000. Freshwater algae of the Southeastern United States. 7. Pigmented Euglenophyceae. Biblioteca Phycologica 106: 1-134. 
Dunlap, J.R., Walne, P.L. \& Kivic, P.A. 1986. Cytological and taxonomic studies of the Euglenales. II. Comparative microarchitecture and cytochemistry of envelopes of Strombomonas and Trachelomonas. British Phycological Journal 21: 399-405.

Franceschini, I.M. 1992. Algues d'eau douce de Porto Alegre, Brésil (les Diatomophycées exclues). Bibliotheca Phycologica 92: 1-72.

Garrels, R. \& Mackenzie, F. 1971. Evolution of sedimentary rocks. W.W. Norton and Company, New York.

Hamm, A. 1969. Die Ermittlung der Gewässergüteklassen bei Flißgewäsern nach dem Gewässergütsystem und Gewässegutenomogramm. Münchner Beiträge zur Abwasser Fischrei und Flußbiologie 15: 46-48.

Hammer, Ø., Harper, D.A.T., Ryan \& P.D. 2001. PAST: Paleontological Statistics Software Package for Education and Data Analysis. Palaeontologia Electronica 4: 1-9.

Hoek, C. van den, Mann, D.G. \& Jahns, H.M. 1995. Algae: in introduction to phycology. Cambrige University Press, Cambridge.

Huber-Pestalozzi, G. 1955. Euglenophyceen. In: G. HuberPestalozzi (ed.). Das phytoplankton des Susswässers: Systematik und Biologie. E. Schweizerbart'sche Verlangsbuchhandlug, Stuttgart 16: 1-605.

Huszar, V.L.M., Menezes, M. \& Fernandes, V.O. 1989. Fitoplâncton de rede da Lagoa Cabiúnas, Macaé, Estado do Rio de Janeiro, Brasil: Uma contribuição a seu conhecimento. Boletim do Museu Nacional 78: $1-43$.

Jati, S. \& Train, S. 1994. Euglenaceae pigmentadas de ambientes lênticos da Ilha Porto Rico, município de Porto Rico, Paraná, Brasil. Iheringia, série Botânica 45: 117-142.

Keppeler, E.C., Lopes, M.R.M. \& Lima, C.S. 2002. Ficoflórula do lago Amapá em Rio Branco - Acre, I: Euglenophyceae. Revista Brasileira de Biologia 59(4): 679-686.

Linton, E., Nudelman, A., Conforti, V. \& Triemer, R.E. 2000. A molecular analyses of the euglenophytes using small subunit rDNA Journal of Phycology 36:740-746.

Ludwig, J.A. \& Reynolds, J.F. 1988. Statistical ecology. A primer on methods and computing. New York: John Wiley \& Sons, Inc. 337p.

Margalef, R. 1983. Limnologia. Omega, Barcelona.

Marin, B., Palm, A.O., Klingberg, M. \& Melkonian, M. 2003. Phylogeny and taxonomic revision of plastidcontaining Euglenophytes based on SSU rDNA Sequence comparisons and synapomorphic signatures in the SSU rRNA Secondary Struture. Protist 154: 99-145.
Menezes, M. 1992. Taxonomic studies on the Strombomonas verrucosa complex (Euglenophyta). Revista Brasileira de Biologia 52: 449-459.

Menezes, M. \& Fernandes, V.O. 1987. Euglenaceae (Euglenophyceae) pigmentadas do município de Cáceres e arredores, Mato Grosso, Brasil: Uma contribuição a seu conhecimento. Rickia 14: 53-71.

Menezes, M. \& Fernandes, V.O. 1989. Euglenaceae (Euglenophyceae) pigmentadas do noroeste do Estado do Mato Grosso, Brasil: municípios de Barra dos Bugres, Cáceres, Juína e Porto Esperidião. Hoehnea 16: 35-55.

Pérez, M.D.C. 2002. Fitoplancton del rio Negro, Uruguai. Limnetica 21: 81-92.

Popova, T.G. 1966. Evglenovyje vodoroski vyp. 1. Flora sporovych rastenijj Izd. Nauka, Moscou.

Norton, T.A., Melkonian, M. \& Andersen, R.A. 1996. Algal biodiversity. Phycologia 35: 308-326.

Round, F.E. 1983. Biologia das algas. 2 ed. Guanabara Dois, Rio de Janeiro.

Shi, Z., Wang, Q., Xie, S., Dai, J. \& Chen, L. 1999. Euglenophyta. (s.1.) il. Flora Algarum Sinicarum Aquae Dulcis, t. 6, Science Press, Beijing.

Sládecék, V. 1973. System of water quality from the Biological Point of View. Archiv für Hydrobiologie 7: 1-218.

Starmach, K. 1983. Euglenophyta. In: K. Starmach (ed.). Flora Slodkowodna Polski, v.3. Polska Academia Nauk, Warszawa.

Tell, G. \& Conforti, V.T.D. 1986. Euglenophyta pigmentadas de la Argentina. Biblioteca Phycologica 75: 1-301.

Tell, G. \& Conforti, V.T.D. 1988. Quelques Strombomonas Defl. ( Euglenophyta) de l'Argentina au microscope photonique et életronique à balayage. Nova Hedwigia 46: 541-556.

Train, S.T. \& Rodrigues, L.C. 2004. Phytoplanktonic Assemblages. In: S.M., Thomaz, A.A., Agostinho, \& N.S., Hahn (orgs.) The upper Paraná River and its Floodplan: Physical aspects, Ecology and Conservation. Backhuys Publishers, Netherlands, pp. 103-124.

Xavier, M.B. 1988. O gênero Strombomonas Deflandre de lagos do Parque Estadual das Fontes do Ipiranga, São Paulo, Brasil. pp.473-505. In: J.G. Tundisi (ed.). Limnologia e Manejo de Represas. EESC/USP/ CRHEA/ACIESP, São Paulo.

Xavier, M.B. 1993. Distribuição vertical das Euglenaceae pigmentadas do Rio Grande, Represa Billings, São Paulo, Brasil. Acta limnológica Brasiliensia 6: 11-30.

Xavier, M. B. 1994. Criptógamos do Parque Estadual das fontes do Ipiranga, São Paulo, SP. Algas, 5: Euglenaceae pigmentadas), Hoehnea 2: 47-73. 
Xavier, M.B. 1996. Fitoplâncton do Rio Grande, Represa Bilings, São Paulo, Brasil: estudo taxonômico (19851996). Iheringia, série Botânica 47: 103-122.

Wang, Q.X., Liu, H.-J., Yu, J., Sun, S.-Q., Zhang, D.W., \& Bao, W.-M. 2003. Comparative studies on the fine struture and elemental composition of envelopes of Trachelomonas and Strombomonas (Euglenophyta). Acta Botanica Sinica 45: 601-607.
West, L.K. \& Walne, P.L. 1980. Trachelomonas hispida var. coronata (Euglenophyceae). III. Envelope elemental composition and mineralization. Journal of Phycology 16: 583-591.

Wolowski, K. \& Walne, P.L. 2007. Strombomonas and Trachelomonas species (Euglenophyta) from south-eastern USA, Eur. Journal of Phycololgy 42: 409-431. 